\title{
Investigation of Langdon effect on the stimulated backward Raman and Brillouin scattering
}

\author{
Jie Qiu, Liang Hao *, Li Hua Cao, and Shiyang Zou \\ Institute of Applied Physics and Computational Mathematics, Beijing, 100094, China
}

(Dated: August 3, 2021)

\begin{abstract}
In a laser-irradiated plasma, the Langdon effect makes the electron energy distribution function (EEDF) tend to a super-Gaussian distribution, which has important influences on laser plasma instabilities. In this work, the influences of a super-Gaussian EEDF on the convective stimulated backward Raman scattering (SRS) and stimulated backward Brillouin scattering (SBS) are studied systematically for a wide range of typical plasma parameters in the inertial confinement fusion (ICF). Distinct behaviors are found for SRS and SBS in the variation trend of the peak spatial growth rate and the corresponding wavelength of the scattered light. Especially, the Langdon effect on the SBS in plasmas with different ion species and isotopes is analyzed in detail, and the parameter boundary for judging the variation trend of the peak spatial growth rate of SBS with the superGaussian exponent is presented for the first time. In certain plasma parameter region, it is found that the Langdon effect could enhance SBS in mixed plasma, which may attenuate the improvement in suppressing SBS by mixing low-Z ions into the high-Z plasma. These comprehension of Langdon effect on LPIs would contribute to a better understanding of SRS and SBS in experiments.
\end{abstract}

PACS numbers: 52.50Gi, 52.65.Rr, 52.38.Kd

\section{INTRODUCTION}

In laser-driven inertial confinement fusion (ICF), the dominant heating mechanism is inverse bremsstrahlung heating. When the heating rate of inverse bremsstrahlung exceeds the electron thermalization rate, the electron energy distribution function (EEDF) would deviate from a Maxwellian EEDF and become a super-Gaussian one [1, 2]. This so-called Langdon effect is tightly related to laser intensity $I_{0}$, electron temperature $T_{\mathrm{e}}$ and charge state $Z$ of plasma, and has important influences on the process of electron conduction, inverse bremsstrahlung absorption, laser plasma instabilities. Recent study showed that with the increasing intensity, the Langdon effect occurred when $Z v_{\text {os }}^{2} / v_{\text {the }}^{2} \gg 1$ but would be suppressed by the nonlinear effect of high laser intensity when $v_{\mathrm{os}} \gg v_{\text {the }}$, where $v_{\mathrm{os}}$ and $v_{\text {the }}$ are the electron quiver velocity and electron thermal velocity respectively [3]. In ICF, the Langdon effect should be important in high-Z plasma ablated from the hohlraum wall in indirect-drive experiments. Besides, recent experimental results diagnosed the presence of super-Gaussian EEDF when multibeams irradiate at the low-Z gas plasma target [4] via its influence on the spectrum of Thomson scattering $[5,6]$. The power transfer of the crossed beams was found to be overestimated by the standard Maxwellian calculations, and the impact of Langdon effect on the crossed beam energy transfer (CBET) was considered to be a possible reason for the discrepancy between the Maxwellian calculations and experimental results on NIF facility [4].

Besides CBET, backward stimulated Raman scatter-

*Corresponding author: hao_liang@iapcm.ac.cn ing (SRS) and backward simulated Brillouin scattering (SBS) are also important laser plasma instabilities (LPIs) in ICF. Commonly, SRS and SBS are three-wave interaction processes where an incident electromagnetic wave (EMW) decays into a backscattered EMW and a forward propagating electron plasma wave (EPW) or ion acoustic wave (IAW), respectively. They are detrimental to inertial confinement fusion, since the backscattered light can take energy away from the incident laser, and the hot electrons generated by SRS can preheat the capsule [7]. Besides, the strong backscattered light of SBS also has a potential risk to damage the optical device within the laser facility. In experiments, the spectra and reflectivity of the backscattered light are important diagnostics for SRS and SBS. Currently, the investigation of the scattered spectra and reflectivity usually assumes a Maxwellian EEDF [8, 9]. However, there was some discrepancy between the experimentally measured SRS spectra and the simulated result by the ray-tracing method [10, 11]. To match the experimental reflectivity of SRS, an artificial seed of SRS backscattered light was also needed in calculations [11]. Because the SRS and SBS are very sensitive to the EEDF, one possible source of the discrepancy is the assumption of the Maxwellian EEDF in current physics model of the raytracing method. Although the impact of super-Gaussian EEDF on the threshold of SRS and the ion acoustic frequency of SBS were studied for some specific plasma conditions $[12,13]$, for practical interest, it is necessary to study the effects of super-Gaussian EEDF on the gain and spectra of SRS and SBS systematically for a wide range of typical plasma conditions and plasma compositions in ICF.

To help resolve these issues, in this work, the influences of super-Gaussian EEDF with different exponents on the SRS and SBS for a wide range of plasma conditions are 
studied systematically based on the linear gain coefficient calculation. Besides the distinct behaviors in wavelength of scattered light and the growth rate of SRS and SBS, some of which are consistent with previous works, the effects of super-Gaussian EEDF on the peak value of spatial grow rate of SBS are found to be different in plasma with different ion compositions. For each typical plasma composition, the boundary of plasma parameters for the variation trend of the peak spatial growth rate of SBS with the super-Gaussian exponent is presented for the first time, which is useful for the convenient judgement of the variation trend of SBS. One interesting result is that the Langdon effect can weaken SBS in single species plasma but enhance SBS in mixed plasma under certain plasma parameter conditions. For such parameters, although mixing low-Z ion species is often used to suppress SBS $[14,15]$, Langdon effect may attenuate this improvement. Furthermore, it is also found that the isotopic type of the low-Z mixture can have a significant impact on SBS. This work not only advances the further physical modeling and ray-tracing simulations of backscattering instabilities with the consideration of Langdon effect, but also helps to understand the spectra and growth of SRS and SBS in experiments better.

This paper is organized as follows: In Section II, the theoretical analysis method is specified. In Section III, the influence of a super-Gaussian EEDF on the linear gain coefficient versus wavelength is discussed for SRS and SBS processes under various plasma conditions. In Section IV, the conclusions as well as some discussions are given.

\section{THEORETICAL ANALYSIS METHOD}

Due to the inverse Bremsstrahlung (IB) Langdon heating effect in a laser-irradiated plasma, the slow-variation component of the electron energy distribution function (EEDF) can deviate from the Maxwellian [1]. This distorted EEDF can be described by a super-Gaussian function [2],

$$
f_{\mathrm{e} 0}(v)=\frac{n_{\mathrm{e}} m}{4 \pi v_{\mathrm{the}}^{3} \beta_{m}^{3} \Gamma(3 / m)} \exp \left[-\left(\frac{v}{\beta_{m} v_{\mathrm{the}}}\right)^{m}\right],
$$

where the distortion is characterized by the super-Gaussian exponent $m$, the factor $\beta_{m}=$ $\sqrt{3 \Gamma(3 / m) / \Gamma(5 / m)}, \quad \Gamma$ is the gamma function, and $v_{\text {the }}=\sqrt{T_{\mathrm{e}} / m_{\mathrm{e}}}$ is the electron thermal velocity. The super-Gaussian exponent $m$ is determined by the competition between Langdon heating and electron-electron collisions. By fitting to the Fokker-Planck simulation, a formula of $m$ which is valid from low to very high intensity is given as [3]

$$
m=2+\frac{3}{1+0.62 / \alpha_{\text {Ln }}},
$$

where $\alpha_{\text {Ln }}=Z_{\text {eff }}\left(v_{\text {os }}^{2} / v_{\text {the }}^{2}\right) /\left(v_{\text {os }}^{2} / v_{\text {the }}^{2}+1\right)^{2}, Z_{\text {eff }}=\overline{Z^{2}} / \bar{Z}$, and $v_{\mathrm{os}} \equiv e E_{0} / m_{\mathrm{e}} \omega_{0}$ is the electron quiver velocity with
$E_{0}$ and $\omega_{0}$ as the amplitude and frequency of the incident laser, respectively.

As resonant instabilities, SRS and SBS are sensitive to the EEDF and background plasma parameters. To investigate the impact of super-Gaussian EEDF on SRS and SBS with different $m$ and plasma parameters, we start from the linear gain exponent which is an important quantity to characterize the convective amplification of SRS and SBS [7] and its spectrum had been widely used to calculate the backscattered SRS and SBS spectra via the ray-tracing method [9]. In WKB approximation, the spectrum of linear gain exponent is defined as

$$
\mathrm{G}_{\mathrm{R}, \mathrm{B}}\left(\omega_{\mathrm{s}}\right)=\int_{\text {path }} \mathrm{K}_{\mathrm{R}, \mathrm{B}}\left(z, \omega_{\mathrm{s}}\right) d z,
$$

where the subscript R or B denotes the SRS or SBS process, and $\omega_{s}$ is the radian frequency of the scattered light. The integration is along one specified laser ray, and the local spatial growth rate $K_{R, B}$ obtained by the kinetic theory [16] is given by,

$$
\mathrm{K}_{\mathrm{R}, \mathrm{B}}\left(\omega_{\mathrm{s}}\right)=\frac{1}{4} \frac{k_{1, a}^{2} v_{\mathrm{os}}^{2}}{k_{\mathrm{s}} c^{2}} \operatorname{Im}\left[\frac{\chi_{\mathrm{e}}\left(1+\chi_{\mathrm{ion}}\right)}{\epsilon}\right]
$$

where the subscript 1 or $a$ is for EPW or IAW respectively, $\chi_{\mathrm{e}}\left(\omega_{\mathrm{l}, a}, k_{\mathrm{l}, a}\right)$ is the electron susceptibility, $\chi_{\mathrm{ion}}\left(\omega_{\mathrm{l}, a}, k_{\mathrm{l}, a}\right)$ is the total susceptibility of ions, and $\epsilon=1+\chi_{\mathrm{e}}+\chi_{\text {ion }}$ is the dielectric function. The frequency $\omega_{\mathrm{s}}$ and wavenumber $k_{\mathrm{S}}$ for the scattered wave are related to $\omega_{\mathrm{l}, a}$ and $k_{1, a}$ for EPW or IAW by the frequency and wave-number matching condition

$$
\begin{aligned}
\omega_{0} & =\omega_{\mathrm{s}}+\omega_{1, \mathrm{a}} \\
k_{0} & =k_{\mathrm{s}}+k_{\mathrm{l}, \mathrm{a}}
\end{aligned}
$$

where $k_{0}$ is the wavenumber of the incident laser. The incident laser and scattered light satisfy the dispersion relation for the EMWs,

$$
\omega_{0, \mathrm{~s}}^{2}=\omega_{\mathrm{pe}}^{2}+c^{2} k_{0, \mathrm{~s}}^{2} .
$$

The linear kinetic electron susceptibility is obtained from the perturbed Vlasov equation [17]

$$
\begin{aligned}
& \frac{\partial \delta f_{\mathrm{e}}}{\partial t}+\mathbf{v} \cdot \nabla \delta f_{\mathrm{e}}=\frac{e \mathbf{E}}{m} \cdot \frac{\partial f_{\mathrm{e}}}{\partial \mathbf{v}} \\
& \delta n_{\mathrm{e}}=\int \delta f_{\mathrm{e}} d \mathbf{v}=\left(\chi_{\mathrm{e}} / e\right) \epsilon_{0} \nabla \cdot \mathbf{E}
\end{aligned}
$$

where $f_{\mathrm{e}}$ is the EEDF of the background plasma, $\delta f_{\mathrm{e}}$ and $\delta n_{\mathrm{e}}$ are the perturbation of EEDF and electron density respectively, $\mathbf{E}$ is the electrostatic field, $e$ is the charge of electron, and $\epsilon_{0}$ is the vacuum permittivity. Notice that the background EEDF includes both a slow-variation component $f_{\mathrm{e} 0}$ and the high frequency components such as $f_{\mathrm{e} 1}$ which oscillates at $\omega_{0}$. However, only $f_{\mathrm{e} 0}$ can participate in the generation of EPW or IAW with both the perturbation $\delta n_{\mathrm{e}}$ and $\mathbf{E}$ at frequency $\omega_{\mathrm{l}, \mathrm{a}}$, since the Gauss's law $\epsilon_{0} \nabla \cdot \mathbf{E}=-e \delta n_{\mathrm{e}}+\sum Z_{i \alpha} \delta n_{\mathrm{i} \alpha}$, which requires 
resonance of $\mathbf{E}$ and $\delta n_{\mathrm{e}}$, can not be satisfied for the density perturbation at the frequency $\omega_{0} \pm \omega_{1, \text { a }}$ driven by $\mathbf{E}$ and the high frequency component $f_{\mathrm{e} 1}$. In practical ray-tracing simulation, the main pulse are divided into different time slices with interval such as 100ps $\left(\sim 10^{5}\right.$ laser period), and the gain $\mathrm{G}_{\mathrm{R}, \mathrm{B}}$ calculated at an instantaneous step within each time slice is used to evaluate the average level of SRS and SBS in the corresponding time slice $[8,10,11]$. Consequently, $f_{\mathrm{e}}$ can be substituted by $f_{\mathrm{e} 0}$ which is assumed to be constant within each time slice in calculating $\chi_{\mathrm{e}}$. And

$$
\begin{aligned}
\chi_{\mathrm{e}}(\omega, k) & =\frac{\omega_{\mathrm{pe}}^{2}}{k^{2} \int f_{\mathrm{e} 0} d \mathbf{v}} \int \frac{\mathbf{k} \cdot \partial f_{\mathrm{e} 0} / \partial \mathbf{v}}{\omega-\mathbf{k} \cdot \mathbf{v}} \mathrm{d} \mathbf{v} \\
& =\frac{\omega_{\mathrm{pe}}^{2}}{k^{2} \int_{-\infty}^{\infty} f_{\mathrm{e} 0}^{x} d v_{x}} \int_{-\infty}^{\infty} \frac{\partial f_{\mathrm{e} 0}^{x} / \partial v_{x}}{\omega / k-v_{x}} d v_{x}
\end{aligned}
$$

where $\omega_{\text {pe }}$ is the electron plasma frequency, and the $1 \mathrm{D}$ distribution function $f_{\mathrm{e} 0}^{x}\left(v_{x}\right)=\iint f_{\mathrm{e} 0}\left(v_{x}, v_{y}, v_{z}\right) d v_{y} d v_{z}$. For the isotropic super-Gaussian EEDF given in Eq. (1), it can be obtained

$$
\chi_{\mathrm{e}}(\omega, k, m)=\frac{1}{k^{2} \lambda_{\text {De }}^{2}} \mathcal{Z}_{\mathrm{e}}\left[\frac{\omega}{k v_{\text {the }}}, m\right]
$$

where

$$
\mathcal{Z}_{\mathrm{e}}[x, m] \equiv \frac{1}{A_{m}}\left[1+\frac{m}{2 \Gamma(1 / m)} \frac{x}{\beta_{m}} \int_{-\infty}^{\infty} \frac{\exp \left(-u^{m}\right)}{u-\frac{x}{\beta_{m}}} d u\right]
$$

where $A_{m}=3 \Gamma^{2}(3 / m) /[\Gamma(1 / m) \Gamma(5 / m)]$.

A Maxwellian distribution with an ion temperature $T_{\mathrm{i}}$ is assumed for all the ion species, yielding the ion susceptibility,

$$
\chi_{\mathrm{ion}}=\sum_{\alpha} \chi_{\mathrm{i} \alpha}(\omega, k)=\sum_{\alpha} \frac{1}{k^{2} \lambda_{\mathrm{D} \alpha}^{2}}\left[1+\zeta_{\alpha} \mathcal{Z}\left(\zeta_{\alpha}\right)\right]
$$

summed over the ion species $\alpha$, where $\mathcal{Z}\left(\zeta_{\alpha}\right)$ is the plasma dispersion function with $\zeta_{\alpha} \equiv \omega / \sqrt{2} v_{\text {th } \alpha} k$, the thermal velocity $v_{\text {th } \alpha}=\sqrt{T_{\mathrm{i}} / m_{\alpha}}$, the Debye length $\lambda_{\mathrm{D} \alpha}=v_{\mathrm{th} \alpha} / \omega_{\mathrm{p} \alpha}$, and the ion plasma frequency $\omega_{\mathrm{p} \alpha}=$ $\sqrt{e^{2} Z_{\alpha}^{2} n_{\mathrm{i} \alpha} / \epsilon_{0} m_{\alpha}}$ with charge state $Z_{\alpha}$ and ion mass $m_{\alpha}$.

Since the linear gain exponent and the time-resolved spectra of backscattered light of SRS and SBS can be obtained by integrating the spatial growth rate $K_{R, B}$ along one ray path with specified practical profiles of plasma parameters usually provided by the radiation hydrodynamic simulations, as done in the ray-tracing method [8, 9], we mainly analyze the impact of $m$ on $K_{R, B}$ given in Eq. (4) to identify the behaviors under different plasma parameters and plasma composition in following section.

\section{INFLUENCES OF LANGDON EFFECT ON SRS AND SBS}

In ICF hohlraum, the evolution of background plasma is determined by all the irradiating beams. The typi- cal ion species includes the initial filled gas like mid-Z $\mathrm{C}_{5} \mathrm{H}_{12}$ or $\mathrm{CO}_{2}$, or low- $\mathrm{Z} \mathrm{He}$ or the mixture of $\mathrm{H}_{2}$ and $\mathrm{He}$ (labeled as HHe in following paper), the mid-Z $\mathrm{CH}$ plasma from the ablation off the capsule, and the high$\mathrm{Z}$ plasma such as $\mathrm{Au}$ or $\mathrm{AuB}$ ablated from hohlraum wall $[7,14,15]$. According to the empirical formula given by Eq. (2), the super-Gaussian exponent $m$ depends on $Z_{\text {eff }}$ and $v_{\mathrm{os}}^{2} / v_{\text {the }}^{2} \propto I_{15} / T_{\mathrm{e}, \mathrm{KeV}}\left(1-n_{\mathrm{e}} / n_{\mathrm{c}}\right)^{1 / 2}$, where $I_{15}=I_{0}\left[\mathrm{~W} / \mathrm{cm}^{2}\right] / 10^{15}, T_{\mathrm{e}, \mathrm{KeV}}$ is the value of $T_{\mathrm{e}}$ in unit of $\mathrm{KeV}$, and $n_{\mathrm{c}}$ is the critical density of incident laser with wavelength $\lambda_{0}=0.351 \mu \mathrm{m}$ in this paper. In hohlraum experiment, the typical $T_{\mathrm{e}}$ is about $\sim \mathrm{KeV}$. Considering the complex beam overlapping [18] and the high intensity speckles in the laser spot [7], the local intensity for IB heating can range from $10^{14}$ to about $10^{16} \mathrm{~W} / \mathrm{cm}^{2}$. Consequently, the possible values of $I_{15} / T_{\mathrm{e}, \mathrm{KeV}}\left(1-n_{\mathrm{e}} / n_{\mathrm{c}}\right)^{1 / 2}$ approximately cover the range of 0.1-10 in ICF hohlraum, over which the variation of $m$ with $I_{15} / T_{\mathrm{e}, \mathrm{KeV}}\left(1-n_{\mathrm{e}} / n_{\mathrm{c}}\right)^{1 / 2}$ is shown in Fig. 1 for the typical ion compositions in hohlraum. It can be seen that the typical value of the super-Gaussian exponent $m$ can vary from 2 to about 3, 4 and 5 for the low-Z, mid-Z and high-Z plasma, respectively. Consequently, although the plasma parameters vary at different locations on different rays in hohlraum, the Langdon effect should be prevalent and important to LPIs.

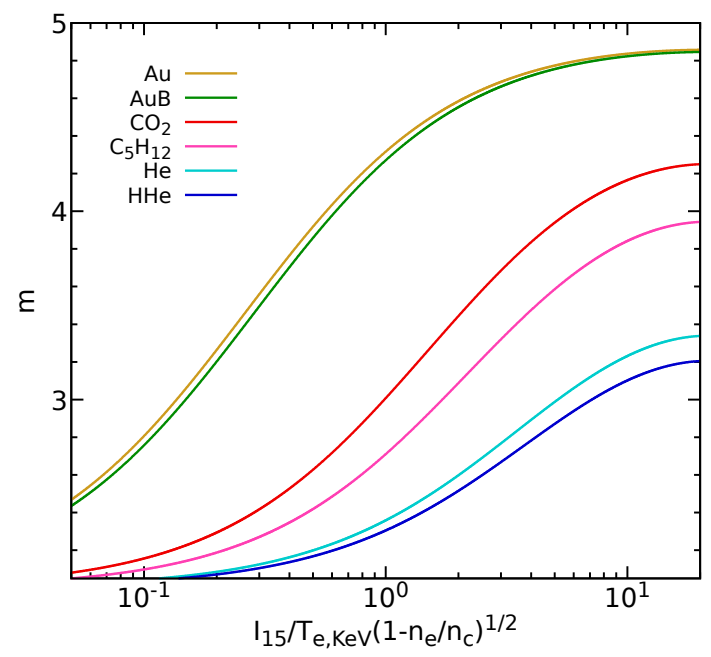

FIG. 1: The variation of $m$ with $I_{15} / T_{\mathrm{e}, \mathrm{KeV}}\left(1-n_{\mathrm{e}} / n_{\mathrm{c}}\right)^{1 / 2}$ for $\mathrm{He}(Z=2)$, $\mathrm{HHe}\left(Z_{\mathrm{eff}} \approx 1.67\right), \mathrm{C}_{5} \mathrm{H}_{12}\left(Z_{\mathrm{eff}} \approx 4.57\right), \mathrm{CO}_{2}$ $\left(Z_{\text {eff }} \approx 7.45\right), \mathrm{Au}(Z=50)$, and $\mathrm{AuB}\left(Z_{\mathrm{Au}}=50, Z_{\mathrm{B}}=5\right.$, $\left.Z_{\text {eff }} \approx 45.9\right)$ plasma in practical parameter range of hohlraum experiment.

\section{A. SRS process}

In hohlraum, SRS mainly occurs in the gas region with low-Z or mid-Z plasma [7], for which $m$ can vary from 2 to about 4 as shown in Fig. 1. Through a systematic analysis of the influence of $m$ on $K_{\mathrm{R}}$ for various 
plasma conditions, it is found that the wavelength of SRS backscattered light corresponding to the peak value of $\mathrm{K}_{\mathrm{R}}$ decreases with $m$ in regime of high $n_{\mathrm{e}}$ and low $T_{\mathrm{e}}$ while increases with $m$ in regime of low $n_{\mathrm{e}}$ and high $T_{\mathrm{e}}$, but the peak value of $\mathrm{K}_{\mathrm{R}}$ always increases with $m$, irrespective of the specific plasma condition. Besides, the half width of $K_{R}$ versus $\lambda_{R}$ is anti-correlated to the peak value of $K_{R}$, implying that it always decreases with $m$. To illustrate these effects, as two typical examples in different regimes of $n_{\mathrm{e}}$ and $T_{\mathrm{e}}$, case I and case II are shown in Fig. 2(a) and Fig. 2(b), respectively. Notice that $K_{R}$ is proportional to the intensity of single beam which stimulates the SRS, while the single beam intensity may not be the total intensity leading to the IB heating, due to effects such as beam overlapping. For the universality, $\mathrm{K}_{\mathrm{R}} / I_{15}$ which is independent of the single beam intensity is used to characterize the physics trends in these figures.
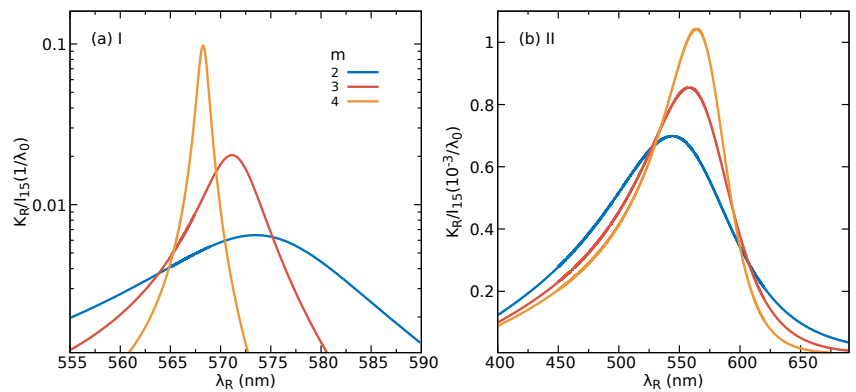

FIG. 2: The spatial growth rate $\left(\mathrm{K}_{\mathrm{R}} / I_{15}\right)$ versus scattered wavelength $\left(\lambda_{\mathrm{R}}\right)$ at different $m$ for (a) case I and (b) case II. In case I, $n_{\mathrm{e}}=0.1 n_{\mathrm{c}}, T_{\mathrm{e}}=3 \mathrm{KeV}$. In case II, $n_{\mathrm{e}}=0.05 n_{\mathrm{c}}$, $T_{\mathrm{e}}=5 \mathrm{KeV}$.

Commonly, $K_{\mathrm{R}}$ peaks approximately at the naturally resonant frequency of EPW, which satisfies the dispersion equation $\chi_{\mathrm{e}}\left(\omega_{\mathrm{l}}-\dot{\mathrm{i}} \nu_{1}, k_{\mathrm{l}}, m\right)+1=0$, where $k_{\mathrm{l}}, \omega_{\mathrm{l}}$ and $\nu_{1}$ are the wavenumber, frequency and Landau damping of EPW, respectively. And the wavelength of SRS scattered light is related to $\omega_{\mathrm{l}}$ by $\lambda_{\mathrm{R}}=\omega_{0} \lambda_{0} /\left(\omega_{0}-\omega_{1}\right)$. To understand the reason for the different variation trends of peak wavelength with $m$ at different plasma conditions, a normalized dispersion relation

$$
\mathcal{Z}_{\mathrm{e}}\left[\frac{\omega_{1}-\dot{\mathrm{n}} \nu_{1}}{\omega_{\mathrm{pe}}\left(k_{1} \lambda_{\mathrm{De}}\right)}, m\right]+k_{1}^{2} \lambda_{\mathrm{De}}^{2}=0
$$

of $\omega_{\mathrm{l}} / \omega_{\text {pe }}$ versus $k_{\mathrm{l}} \lambda_{\text {De }}$ for EPW is calculated at different $m$ as shown in Fig. 3. For a given $k_{1} \lambda_{\text {De }}$, there are roughly three regimes in Fig. 3(a). In regime I, where $k_{1} \lambda_{\text {De }} \lesssim 0.43, \omega_{1}$ decreases monotonously with $m$, and the dependence of the dispersion relation on $m$ is rather weak at about $k_{1} \lambda_{\text {De }}<0.2$. In regime II, where $0.43<k_{1} \lambda_{\text {De }} \lesssim 0.55$, the variation trend of $\omega_{1}$ with $m$ becomes non-monotonous. In regime III, where $k_{1} \lambda_{\text {De }}>0.55, \omega_{1}$ increases monotonously with $m$. In fact, for a fixed $k_{1} \lambda_{\text {De }}, \Delta \omega_{1} / \Delta m=-\left(\partial \mathcal{Z}_{\mathrm{e}} / \partial m\right) /\left(\partial \mathcal{Z}_{\mathrm{e}} / \partial \omega_{\mathrm{l}}\right)$ can be obtained from Eq. (12). It can be proven $\partial \mathcal{Z}_{\mathrm{e}} / \partial \omega_{\mathrm{l}}>0$ at the EPW frequency, but the sign of $\partial \mathcal{Z}_{\mathrm{e}} / \partial m$ depends on the value of $k_{1} \lambda_{\text {De }}$ and $m$, which results in the three regimes of different $k_{1} \lambda_{\text {De }}$. For the case I and case II shown in Fig. 2, by solving the dispersion relations Eq. (6) and Eq. (12) together with the matching conditions Eq. (5), $k_{1} \lambda_{\text {De }}$ can be obtained to be about 0.36 for case I and 0.68 for case II as indicated in Fig. 3(a). As expected, case I and case II are located in the regimes where $\lambda_{\mathrm{R}}$ is blue-shifted and red-shifted with $m$, respectively.
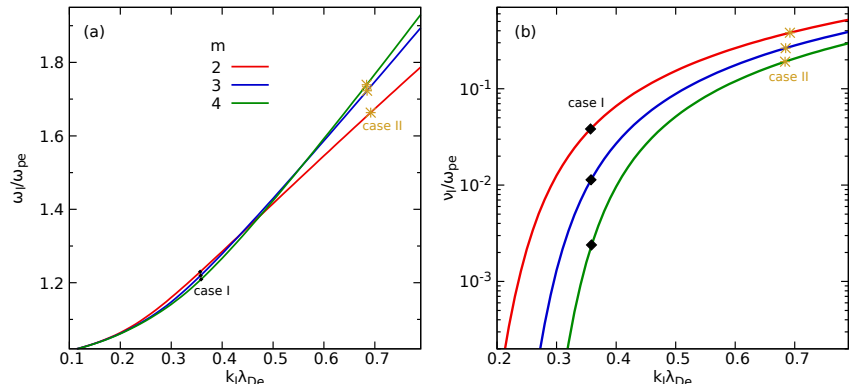

FIG. 3: (a) The dispersion relations and (b) Landau damping of EPW at different $m$. The matching points of case I and case II are indicated by the points for each $m$.

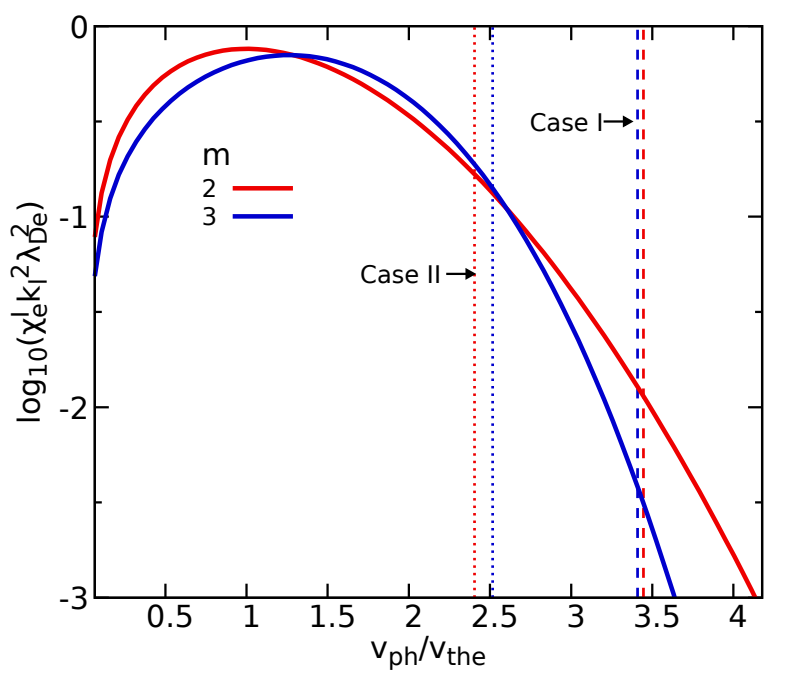

FIG. 4: $\quad \chi_{\mathrm{e}}^{\mathrm{I}} k_{1}^{2} \lambda_{\mathrm{De}}^{2} \propto-\partial f_{\mathrm{e} 0}^{x} / \partial v_{x}$ (see Eq. 14) at different $m$. The matched EPW phase velocities at each $m$ for case I and case II are indicated by the vertical lines (each $m$ with one color).

To understand the reason for the behavior of the peak value of $\mathrm{K}_{\mathrm{R}}$ with $m$ and the anti-correlation between the peak value and the half width of $K_{R}$,

$$
\mathrm{K}_{\mathrm{R}} \propto-\operatorname{Im}\left[\frac{1}{1+\chi_{\mathrm{e}}}\right]=\frac{\chi_{\mathrm{e}}^{\mathrm{I}}}{\left(1+\chi_{\mathrm{e}}^{\mathrm{R}}\right)^{2}+\left(\chi_{\mathrm{e}}^{\mathrm{I}}\right)^{2}}
$$

can be deduced from Eq. (4), where the superscripts R and I denote the real and imaginary parts, respectively. According to Eq. (13), the half width is determined by 
$\left|1+\chi_{\mathrm{e}}^{\mathrm{R}}\right| \approx \chi_{\mathrm{e}}^{\mathrm{I}}$, so a higher peak value of $\mathrm{K}_{\mathrm{R}}$ is always related to a smaller half width of it. At the peak, $\chi_{\mathrm{e}}^{\mathrm{R}}+1 \approx$ 0 , and the peak value of $K_{\mathrm{R}}$ is proportional to $1 / \chi_{\mathrm{e}}^{\mathrm{I}}$. Since $\chi_{\mathrm{e}}^{\mathrm{I}}=\nu_{\mathrm{l}}\left(\partial \epsilon^{\mathrm{R}} / \partial \omega\right) \approx \nu_{\mathrm{l}}\left(\partial \chi_{\mathrm{e}}^{\mathrm{R}} / \partial \omega\right)[9]$, it is $\nu_{\mathrm{l}}$ that primarily determines the trend of the peak value of $K_{\mathrm{R}}$ with $m$. As shown in Fig. 3(b), $\nu_{1}$ decreases with $m$ for all $k_{1} \lambda_{\text {De }}$, so the peak value of $K_{\mathrm{R}}$ always increase with $m$. The trend of Landau damping with $m$ and $k_{1} \lambda_{\text {De }}$ can be understood from the feature of the EEDF near the matched phase velocity $v_{\mathrm{ph}}=\omega_{\mathrm{l}} / k_{\mathrm{l}}$. For $\nu_{\mathrm{l}} \ll \omega_{1}, \chi_{\mathrm{e}}^{\mathrm{I}}$ from Eq. (8) can be written as

$$
\begin{aligned}
\chi_{\mathrm{e}}^{\mathrm{I}} \approx \frac{\omega_{\mathrm{pe}}^{2}}{k_{1}^{2} \int_{-\infty}^{\infty} f_{\mathrm{e} 0}^{x} d v_{x}} & \int_{-\infty}^{\infty} \frac{\partial f_{\mathrm{e} 0}^{x}}{\partial v_{x}} \operatorname{Im}\left[\frac{1}{\omega_{\mathrm{l}} / k_{\mathrm{l}}-v_{x}}\right] d v_{x} \\
& =\left.\frac{-\pi \omega_{\mathrm{pe}}^{2}}{k_{1}^{2} \int_{-\infty}^{\infty} f_{\mathrm{e} 0}^{x} d v_{x}} \frac{\partial f_{\mathrm{e} 0}^{x}}{\partial v_{x}}\right|_{v_{x}=v_{\mathrm{ph}}},
\end{aligned}
$$

where second equality follows by using $\operatorname{Im}[1 /(\omega / k-$ $\left.\left.v_{x}\right)\right]=-\pi \delta\left(\omega / k-v_{x}\right)$ [17]. As shown in Fig. 4, in case I of small $k_{1} \lambda_{\text {De }}$, the matched $v_{\mathrm{ph}}$ is located in the tail of the EEDF where $\chi_{\mathrm{e}}^{\mathrm{I}} \propto e^{-v_{\mathrm{ph}}^{m}}$, and $\nu_{\mathrm{l}}$ decreases with $m$ rapidly. While in case II of large $k_{1} \lambda_{\text {De }}$, the matched $v_{\mathrm{ph}}$ is in the bulk region of the EEDF where $\chi_{\mathrm{e}}^{\mathrm{I}}\left(v_{\mathrm{ph}}\right)$ at different $m$ is close. For such a case, it is the increase of the matched phase velocity with $m$ (see Fig. 3a) that leads to the decrease of Landau damping with $m$.

\section{B. SBS process}

Commonly, SBS can occur in both the gas region with low-Z or mid-Z plasma and the ablated region with high-Z plasma in hohlraum [7]. To illuminate the influence of super-Gaussian exponent $m$ on SBS, several examples with the typical parameters in hohlraum are shown in Figs. 5 and 6 for the low-Z and high-Z plasma with the specific conditions listed in Tables I and II, respectively. In low- $\mathrm{Z} \mathrm{He}$ and $\mathrm{HHe}$ plasma, we take $m=2,2.3,2.6,2.9$ as examples, while in high-Z Au and AuB plasma, $m=2,3,4,4.5$ are discussed. The intensity requirements for the corresponding $m$ of super-Gaussian EEDF, are listed in Tables I and II for these cases, which are possible due to the high intensity speckles and beam overlapping effects. Comparing different cases shown in Figs. 5 and 6 , it is found that the peak wavelength of SBS backscattered light always increases with $m$ for a given parameter conditions and ion composition, but the influence of $m$ on the peak value of $K_{\mathrm{B}}$ is much more complicated. In both $\mathrm{He}$ and $\mathrm{HHe}$ plasmas, the peak value of $\mathrm{K}_{\mathrm{B}}$ can increase or decrease with $m$ according to the plasma conditions. Even at the same condition labeled as case ' $\mathrm{a}$ ', the variation trend of $\mathrm{K}_{\mathrm{B}}$ with $m$ can be opposite in He and HHe plasma. In high-Z case ' $d$ ', the peak $K_{\mathrm{B}}$ decreases with $m$ in pure Au plasma, while the trend of the peak $\mathrm{K}_{\mathrm{B}}$ with $m$ becomes non-monotonic in AuB plasma, and depends sensitively on the isotopic type of the low-Z species B.
TABLE I: The representative cases for $\mathrm{He}$ and $\mathrm{HHe}$ (1:1) plasma. In all cases, the flow velocity is assumed to be zero.

\begin{tabular}{ccccccc}
\hline Case & $n_{\mathrm{e}} / n_{\mathrm{c}}$ & $T_{\mathrm{e}}(\mathrm{KeV})$ & $T_{\mathrm{i}}(\mathrm{KeV})$ & \multicolumn{3}{c}{$I_{15}{ }^{1}$} \\
& & & & $m=2.3$ & $m=2.6$ & $m=2.9$ \\
\hline 'a'-He & 0.08 & 2.5 & 0.5 & 1.9 & 4.8 & 9.8 \\
'b'-He & 0.06 & 2 & 1 & 1.6 & 3.9 & 7.9 \\
'a'-HHe & 0.08 & 2.5 & 0.5 & 2.4 & 6.0 & 12.9 \\
'c'-HHe & 0.06 & 1 & 0.12 & 0.95 & 2.4 & 5.2 \\
\hline
\end{tabular}

${ }^{1}$ The required $I_{15}$ for each $m$ is estimated using Eq. (2).
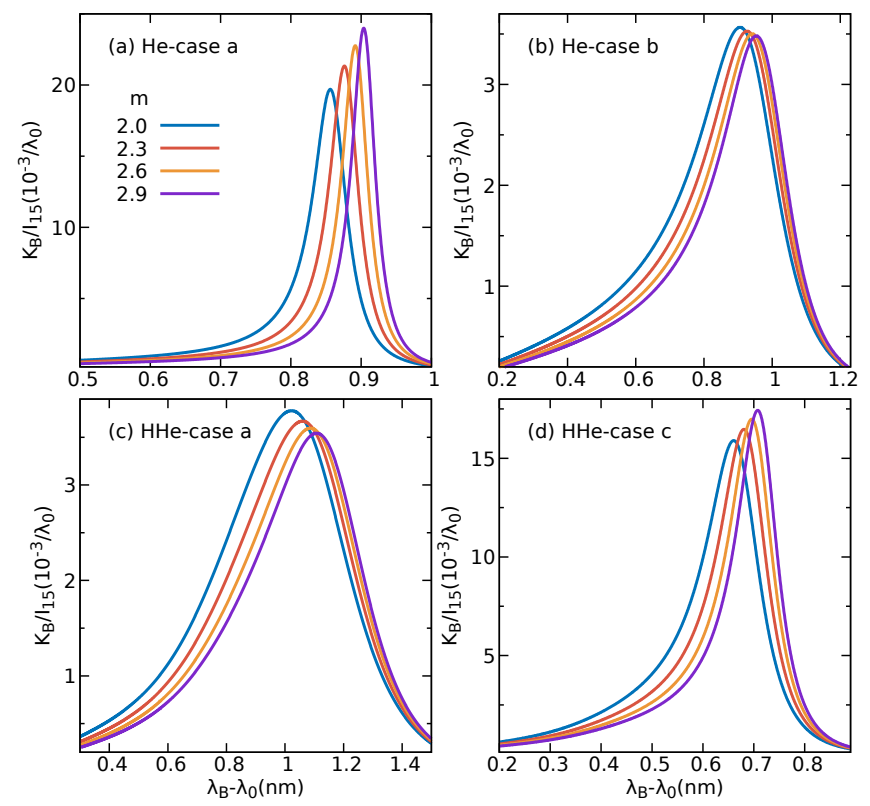

FIG. 5: The spatial growth rate $\left(\mathrm{K}_{\mathrm{B}} / I_{15}\right)$ of SBS versus the scattered wavelength shift $\left(\lambda_{\mathrm{B}}-\lambda_{0}\right)$ at different superGaussian exponents $m$ for several cases in a fully ionized (a-b) $\mathrm{He}$ and (c-d) HHe plasma. The case conditions are specified in Table I.
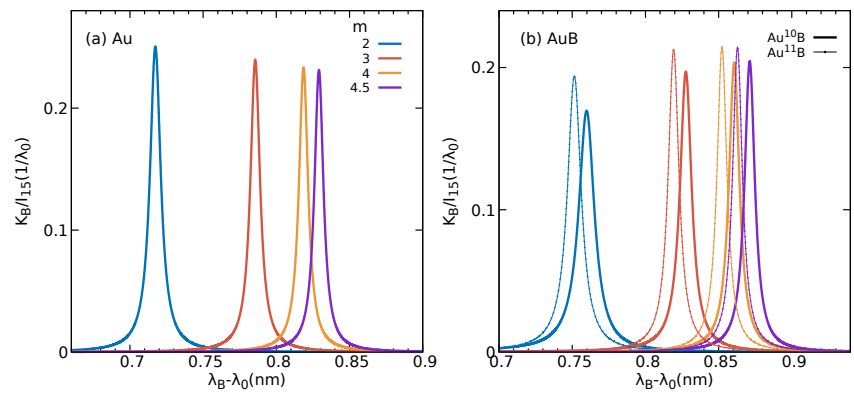

FIG. 6: The convective spatial growth rate $\left(\mathrm{K}_{\mathrm{B}} / I_{15}\right)$ of SBS versus the scattered wavelength shift $\left(\lambda_{\mathrm{B}}-\lambda_{0}\right)$ at different super-Gaussian exponents $m$ in (a) Au plasma and (b) $\mathrm{Au}^{10} \mathrm{~B}$ and $\mathrm{Au}^{11} \mathrm{~B}$ plasmas. The condition of case ' $\mathrm{d}$ ' as specified in Table II is taken.

To comprehend the behavior of $\mathrm{K}_{\mathrm{B}}$ with $m$, Eq. (4) 
TABLE II: The representative cases for $\mathrm{Au}$ and $\mathrm{AuB}$ (1:1) plasma. In all cases, the flow velocity is assumed to be zero.

\begin{tabular}{lccccccc}
\hline Case & \multirow{2}{*}{$Z_{\alpha}$} & \multicolumn{2}{c}{$n_{\mathrm{e}} / n_{\mathrm{c}}$} & $T_{\mathrm{e}}(\mathrm{KeV})$ & $T_{\mathrm{i}}(\mathrm{KeV})$ & \multicolumn{3}{c}{$I_{15}{ }^{1}$} \\
& & & & & $m=3$ & $m=4.5$ \\
\hline 'd'-Au & $Z_{\mathrm{Au}}=50$ & 0.2 & 5 & 0.9 & 0.61 & 2.5 & 6.9 \\
'd'-AuB & $Z_{\mathrm{Au}}=50, Z_{\mathrm{B}}=5$ & 0.2 & 5 & 0.9 & 0.67 & 2.8 & 7.6 \\
\hline
\end{tabular}

${ }^{1}$ The required $I_{15}$ for each $m$ is estimated using Eq. (2).

can be written as

$$
\mathrm{K}_{\mathrm{B}}=-\frac{1}{4} \frac{k_{a}^{2} v_{\mathrm{oS}}^{2}}{k_{\mathrm{S}} c^{2}} \frac{\mathscr{D}^{\mathrm{I}}}{\left(\mathscr{D}^{\mathrm{R}}\right)^{2}+\left(\mathscr{D}^{\mathrm{I}}\right)^{2}},
$$

where

$$
\mathscr{D} \equiv \frac{1}{\chi_{\mathrm{e}}}+\frac{1}{1+\chi_{\text {ion }}}
$$

and superscripts $\mathrm{R}$ and I denote the real and imaginary parts, respectively. Near the peak of $K_{B}, \mathscr{D}^{\mathrm{R}} \approx 0$ is consistent with the dispersion relation of IAW $\epsilon \approx 0$ for weak damping $\left|\chi_{\mathrm{e}}^{\mathrm{I}}\right| \ll\left|\chi_{\mathrm{e}}^{\mathrm{R}}\right|$ and $\left|\chi_{\text {ion }}^{\mathrm{I}}\right| \ll\left|1+\chi_{\text {ion }}^{\mathrm{R}}\right|$, implying $K_{\mathrm{B}}$ peaks near the natural resonance mode. And the peak value of spatial growth rate

$$
\mathrm{K}_{\mathrm{B}} \approx-\frac{1}{4} \frac{k_{a}^{2} v_{\mathrm{oS}}^{2}}{k_{\mathrm{S}} c^{2}} \frac{1}{\mathscr{D}^{\mathrm{I}}}
$$

To see the reason for the redshift of SBS backscattered light with $m$, the dispersion relation of IAW is solved. Under the approximation $\omega_{a} / k_{a} \ll v_{\text {the }}$, for the superGaussian EEDF

$$
\chi_{\mathrm{e}}^{\mathrm{R}} \approx \frac{1}{A_{m} k_{a}^{2} \lambda_{\mathrm{De}}^{2}} \propto \frac{1}{k_{a}^{2}} \frac{n_{\mathrm{e}}}{A_{m} T_{\mathrm{e}}} .
$$

Because $A_{m}$ increases with $m$ monotonically, $\chi_{\mathrm{e}}^{\mathrm{R}}$ decreases with $m$, implying a reduced number of slow electrons to shield the ions due to the Langdon effect [13]. For such $\chi_{\mathrm{e}}^{\mathrm{R}}$, the ion acoustic velocity $c_{\mathrm{s}}=\omega_{a} / k_{a}$ in plasma composed of one or two species $[19,20]$, is given by

$$
c_{\mathrm{s}}^{2}=\frac{1}{2}(A \pm \sqrt{B})
$$

where "+" corresponds to the fast mode, and "-" corresponds to the slow mode, and

$$
A \equiv\left(\frac{\gamma_{1}}{A_{1}}+\frac{\gamma_{2}}{A_{2}}\right) \frac{T_{\mathrm{i}}}{M_{\mathrm{p}}}+\frac{\overline{Z^{2} / A} A_{m} T_{\mathrm{e}}}{\bar{Z} M_{\mathrm{p}} q}
$$

and

$$
\begin{aligned}
& B \equiv\left(\frac{\gamma_{1}}{A_{1}}-\frac{\gamma_{2}}{A_{2}}\right)^{2}\left(\frac{T_{\mathrm{i}}}{M_{\mathrm{p}}}\right)^{2}+\left(\frac{\overline{Z^{2} / A} A_{m} T_{\mathrm{e}}}{\bar{Z} M_{\mathrm{p}} q}\right)^{2} \\
& +2\left(\frac{\gamma_{1}}{A_{1}}-\frac{\gamma_{2}}{A_{2}}\right)\left(\frac{f_{1} Z_{1}^{2}}{A_{1}}-\frac{f_{2} Z_{2}^{2}}{A_{2}}\right) \frac{T_{\mathrm{i}} A_{m} T_{\mathrm{e}}}{M_{\mathrm{p}}^{2} \bar{Z} q} .
\end{aligned}
$$

Here $q \equiv 1+A_{m} k_{a}^{2} \lambda_{\text {De }}^{2}, M_{\mathrm{p}}$ is the proton mass, the ion species is indicated by the subscript $\alpha=1,2, \gamma_{\alpha}$ is the adiabatic exponent, $A_{\alpha}$ is the mass number, and $f_{\alpha}$ is the number fraction of ion species $\alpha$. The bars denote the average, i.e.,

$$
\begin{aligned}
& \bar{Z}=f_{1} Z_{1}+f_{2} Z_{2} \\
& \overline{Z^{2} / A}=f_{1} Z_{1}^{2} / A_{1}+f_{2} Z_{2}^{2} / A_{2} .
\end{aligned}
$$

In the expression of $c_{\mathrm{s}}$, the term $A_{m} T_{\mathrm{e}}$ appears as a whole, so it can be thought that the Debye shielding electron temperature is effectively boosted to $A_{m} T_{\mathrm{e}}$. In Appendix $\mathrm{A}$, it is proven that $c_{\mathrm{s}}$ increases with $A_{m} T_{\mathrm{e}}$ for both the fast and slow modes, when all other parameters such as $T_{\mathrm{i}}$ and $n_{\mathrm{e}}$ are kept constant. So it can be expected that due to the effective increase of Debye shielding electron temperature caused by a super-Gaussian EEDF, the IAW frequency $\omega_{a}=k_{a} c_{\mathrm{s}}$ increases with $m$. Consequently, the scattered wavelength $\lambda_{\mathrm{B}}=\omega_{0} \lambda_{0} /\left(\omega_{0}-\omega_{a}\right)$ increases as $m$ increases.

From Eq. (17), the behavior of the peak value of $K_{B}$ with $m$ is primarily determined by the value of $-\mathscr{D}^{\mathrm{I}}$ near the peak wavelength. Since at the peak $\epsilon \approx 0$ and $\left|\chi_{\mathrm{e}}\right| \approx\left|1+\chi_{\text {ion }}\right|$, it is found $\mathrm{K}_{\mathrm{B}} \propto-1 / \mathscr{D}^{\mathrm{I}} \approx$ $\left|\chi_{\mathrm{e}}\right|\left|1+\chi_{\mathrm{ion}}\right| /\left(\chi_{\mathrm{e}}^{\mathrm{I}}+\chi_{\mathrm{ion}}^{\mathrm{I}}\right)$. Considering that $\chi_{\mathrm{e}}^{\mathrm{I}}+\chi_{\mathrm{ion}}^{\mathrm{I}}=$ $\operatorname{Im}[\epsilon]=\left(\nu_{\mathrm{e}}+\nu_{\text {ion }}\right) \partial \epsilon / \partial \omega$, where $\nu_{\mathrm{e}}$ and $\nu_{\text {ion }}$ are electron and ion Landau damping rates respectively, and $\left|\chi_{\mathrm{e}}\right|\left|1+\chi_{\mathrm{ion}}\right|$ is proportional to the ponderomotive drive for IAW [9], it can be understood that $K_{B}$ is determined by the tradeoff between the ponderomotive drive and the Landau damping of IAW. We can also separate out the ion and electron contributions to $\mathscr{D}^{\mathrm{I}}$,

$$
\mathscr{D}^{\mathrm{I}}=\mathscr{D}_{\text {ion }}^{\mathrm{I}}+\mathscr{D}_{\mathrm{e}}^{\mathrm{I}}
$$

with

$$
\mathscr{D}_{\text {ion }}^{\mathrm{I}} \equiv \operatorname{Im}\left[1 /\left(1+\chi_{\text {ion }}\right)\right]=-\chi_{\text {ion }}^{\mathrm{I}} /\left|1+\chi_{\text {ion }}\right|^{2}
$$

and

$$
\mathscr{D}_{\mathrm{e}}^{\mathrm{I}} \equiv \operatorname{Im}\left[1 / \chi_{\mathrm{e}}\right]=-\chi_{\mathrm{e}}^{\mathrm{I}} /\left|\chi_{\mathrm{e}}\right|^{2} .
$$

At the peak of $\mathrm{K}_{\mathrm{B}}, \mathscr{D}_{\text {ion }}^{\mathrm{I}} / \mathscr{D}_{\mathrm{e}}^{\mathrm{I}} \approx \chi_{\text {ion }}^{\mathrm{I}} / \chi_{\mathrm{e}}^{\mathrm{I}} \approx \nu_{\text {ion }} / \nu_{\mathrm{e}}$, so the relative contribution of electron and ion to $\mathscr{D}^{\mathrm{I}}$ is determined by the relative importance of electron Landau damping versus ion Landau damping. As shown in Fig. 4, the electron Landau damping reaches maximum when the phase velocity $v_{\mathrm{ph}}$ is close to $v_{\text {the }}$. Similarly, the ion damping is maximum when the phase velocity $v_{\mathrm{ph}}$ is 
close to the ion thermal velocity $v_{\mathrm{th} \alpha}$. For SBS, typically $c_{\mathrm{S}} \ll v_{\text {the }}$. When $Z_{\alpha}$ increase, the ion acoustic velocity $c_{\mathrm{S}}$ also increases, making the electron damping $\nu_{\mathrm{e}}$ increase whereas the ion damping $\nu_{\text {ion }}$ decrease when $v_{\text {th } \alpha}<c_{\mathrm{S}}$. The contribution of electron damping or ion damping can be quite different in low- $\mathrm{Z}$ and high- $\mathrm{Z}$ plasma. Furthermore, the possible super-Gaussian exponent $m$ due to Langdon effect is smaller in low-Z plasma than in high-Z plasma. Considering the differences in low-Z plasma and high-Z plasma, in the following, we discuss them separately.
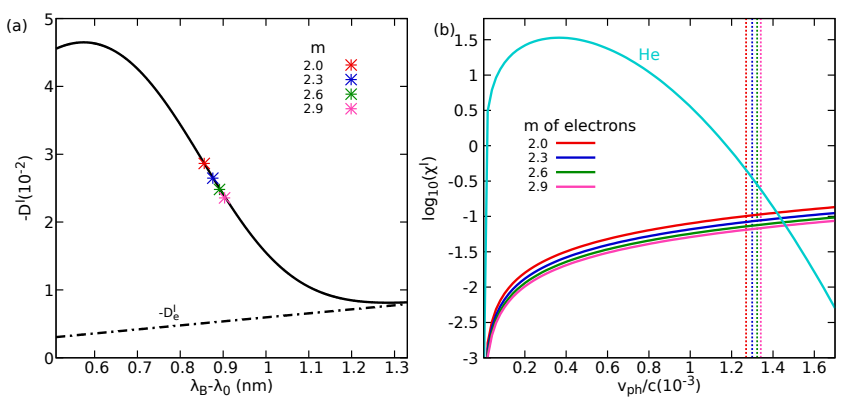

FIG. 7: (a) $-\mathscr{D}^{\mathrm{I}}$ (solid line) and $-\mathscr{D}_{\mathrm{e}}^{\mathrm{I}}$ (dotted-dash line) versus wavelength shift $\left(\lambda_{\mathrm{B}}-\lambda_{0}\right)$ for He plasma. The asterisk symbols indicate the wavelength location corresponding to the peak $\mathrm{K}_{\mathrm{B}}$ at $m=2$ (red), $m=2.3$ (blue), $m=2.6$ (green) and $m=2.9$ (magenta) in case 'a'. (b) $\chi_{\alpha}^{\mathrm{I}} \propto-\partial f_{\alpha}^{x} / \partial v_{x}$ of electrons at different $m$ and He ions versus $v_{\text {ph }}$. The IAW phase velocities corresponding to the peak wavelength for each $m$ in case ' $a$ ' are indicated by the vertical dotted lines.
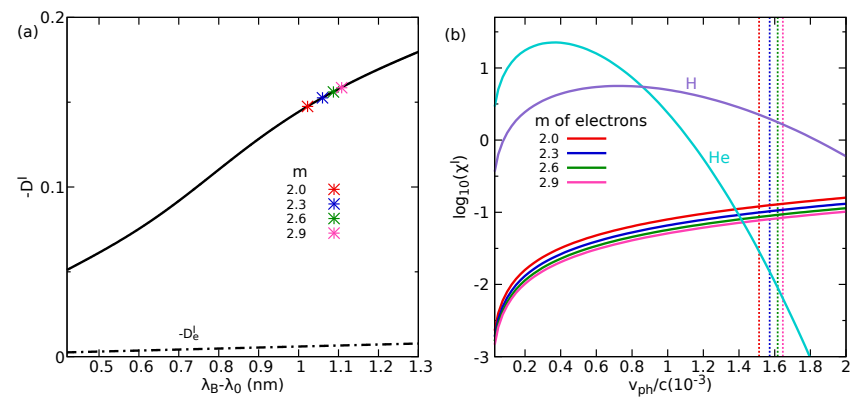

FIG. 8: (a) $-\mathscr{D}^{\mathrm{I}}$ (solid line) and $-\mathscr{D}_{\mathrm{e}}^{\mathrm{I}}$ (dotted-dash line) versus wavelength shift $\left(\lambda_{\mathrm{B}}-\lambda_{0}\right)$ for $\mathrm{HHe}(1: 1)$ plasma. The asterisk symbols indicate the wavelength location corresponding to the peak $\mathrm{K}_{\mathrm{B}}$ at $m=2$ (red), $m=2.3$ (blue), $m=2.6$ (green) and $m=2.9$ (magenta) in case 'a'. (b) $\chi_{\alpha}^{\mathrm{I}} \propto-\partial f_{\alpha}^{x} / \partial v_{x}$ of electrons at different $m, \mathrm{H}$ ions, and $\mathrm{He}$ ions versus $v_{\mathrm{ph}}$. The phase velocities of IAW corresponding to the peak wavelength for each $m$ in case 'a' are indicated by the vertical dotted lines.

In low-Z He and HHe plasmas, taking case 'a' as example, we show $-\mathscr{D}^{\mathrm{I}}$ in Fig. 7(a) and Fig. 8(a) for $\mathrm{He}$ and HHe plasmas, respectively. In proximity to the peak wavelength, $-\mathscr{D}^{\mathrm{I}}$ is mainly contributed by ions, because the ion damping which is proportional to $\chi_{\text {ion }}^{\mathrm{I}}$ is much higher than the electron damping as shown in Figs. 7(b) and $8(\mathrm{~b})$. Since the Maxwellian distribution of ions is assumed, $\mathscr{D}_{\text {ion }}^{\mathrm{I}}$ itself does not depend on $m$. However, since the phase velocity of IAW corresponding to the peak wavelength $\lambda_{\mathrm{B}}$ increases with $m$, the decrease of $-\mathscr{D}_{\text {ion }}^{\mathrm{I}}$ with the peak wavelength $\lambda_{\mathrm{B}}$ at different $m$ in He plasma leads to the increase of the peak value of $\mathrm{K}_{\mathrm{B}}$ with $m$. Similarly in HHe plasma, the increase of $-\mathscr{D}_{\text {ion }}^{\mathrm{I}}$ with the peak wavelength $\lambda_{\mathrm{B}}$ at different $m$ results in the decrease of the peak $\mathrm{K}_{\mathrm{B}}$ with $m$.
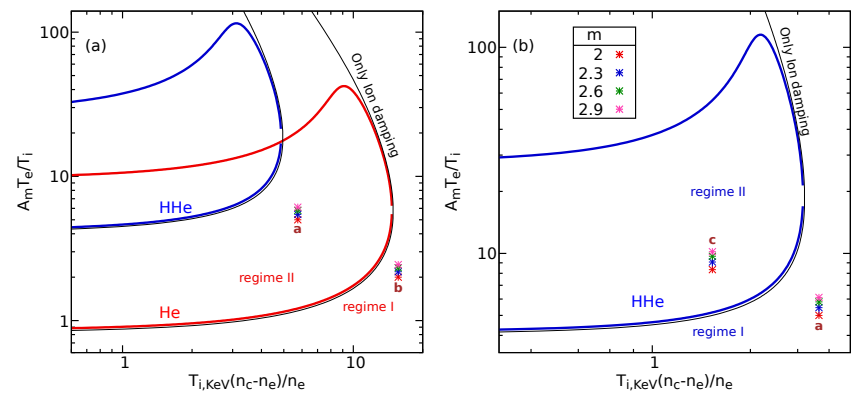

FIG. 9: (a) The boundary between regime I and II as specified by $A_{m} T_{\mathrm{e}} / T_{\mathrm{i}}$ versus $T_{\mathrm{i}}\left(n_{\mathrm{c}}-n_{\mathrm{e}}\right) / n_{\mathrm{e}}$ for He (red line) and HHe (1:1) (blue line) plasmas. (b) Zoomed in boundary for HHe (1:1) (blue line) plasmas. The asterisk symbols indicate different cases with conditions specified in Table I, with the four points corresponding to $m=2, m=2.3, m=2.6$ and $m=2.9$ from bottom to top for each case. The thin black curves show the boundary with ion damping considered only.

It can be concluded that the variation trend of peak $\mathrm{K}_{\mathrm{B}}$ with $m$ is determined by the behavior of $-\mathscr{D}^{\mathrm{I}}$ with $v_{\mathrm{ph}}$ near the phase velocity of IAW corresponding to the peak wavelength. According to this, two parameter regimes can be defined. In regime I, $\partial \mathscr{D}^{\mathrm{I}} / \partial v_{\mathrm{ph}}<0$ near the peak point and thus the peak $\mathrm{K}_{\mathrm{B}}$ decrease with $m$, while $\partial \mathscr{D}^{\mathrm{I}} / \partial v_{\mathrm{ph}}>0$ near the peak point and the peak $\mathrm{K}_{\mathrm{B}}$ increase with $m$ in regime II. So, the boundary between regime I and II satisfies $\partial \mathscr{D}^{\mathrm{I}} / \partial v_{\mathrm{ph}}=0$ at the peak point which maintains $\mathscr{D}^{\mathrm{R}}=0$. [21] These two equations determine a relation between $A_{m} T_{\mathrm{e}} / T_{\mathrm{i}}$ and $T_{\mathrm{i}}\left(1-n_{\mathrm{e}} / n_{\mathrm{c}}\right) / n_{\mathrm{e}}$ for a given ion composition. By numerical solution, the boundary between regime I and II for He and HHe plasma are shown in Fig. 9, where the weak dependence of $-\mathscr{D}_{\mathrm{e}}^{\mathrm{I}}$ on $m$ is ignored, and the different cases shown in Fig. 5 are also labelled as asterisks. As expected, case ' $\mathrm{b}$ ' is located in regime I of He plasma, and case ' $c$ ' is located in regime II of HHe plasma. Case 'a' of $\mathrm{He}$ and HHe are located in the special parameter range that is in regime II of He plasma yet in regime I of HHe plasma, thus the variation trend of peak $\mathrm{K}_{\mathrm{B}}$ with $m$ are opposite in case ' $\mathrm{a}$ ' of $\mathrm{He}$ and HHe plasma even under the same parameter condition.

Now we take a closer look into the case 'a' to comprehend the physical mechanism for the effects of mixture. Since $-\mathscr{D}_{\text {ion }}^{\mathrm{I}}=\chi_{\text {ion }}^{\mathrm{I}} /\left|1+\chi_{\text {ion }}\right|^{2}$ dominates $-\mathscr{D}^{\mathrm{I}}$ near the matched $c_{\mathrm{s}}$ as discussed above, and both $\chi_{\text {ion }}^{\mathrm{I}}$ and $\left|1+\chi_{\text {ion }}\right|^{2}$ drop with $v_{\mathrm{ph}}$, the relative dropping rate of $\chi_{\text {ion }}^{\mathrm{I}}$ and $\left|1+\chi_{\text {ion }}\right|^{2}$ with $v_{\mathrm{ph}}$ determines the sign of 


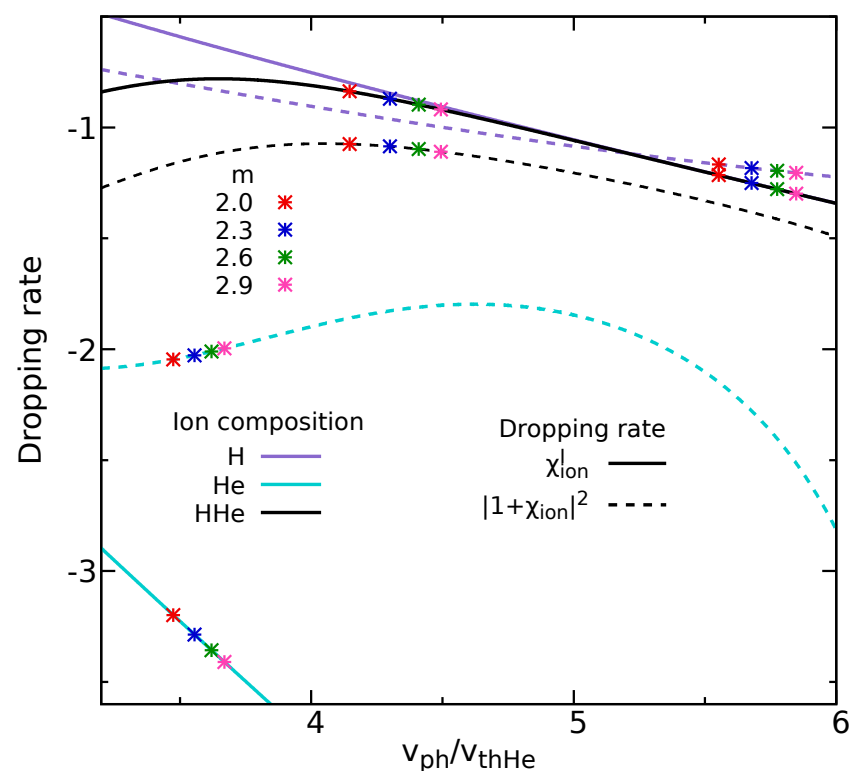

FIG. 10: The dropping rate of $\chi_{\text {ion }}^{\mathrm{I}}\left(\right.$ solid line) and $\left|1+\chi_{\text {ion }}\right|^{2}$ (dashed line) versus $v_{\mathrm{ph}}$ for fully ionized $\mathrm{H}$ (purple), He (blue) and HHe (1:1) (black) plasmas. The asterisk symbols indicate locations of the matched phase velocity corresponding to the peak of $\mathrm{K}_{\mathrm{B}}$ at $m=2$ (red), $m=2.3$ (blue), $m=2.6$ (green) and $m=2.9$ (magenta) in case 'a'.

$\partial \mathscr{D}^{\mathrm{I}} / \partial v_{\text {ph }}$ and thus the regime. In Fig. 10, the dropping rate of $\chi_{\text {ion }}^{\mathrm{I}}$ and $\left|1+\chi_{\text {ion }}\right|^{2}$, which are defined as $\partial\left(\ln \chi_{\text {ion }}^{\mathrm{I}}\right) / \partial v_{\mathrm{ph}}$ and $\partial\left(\ln \left|1+\chi_{\text {ion }}\right|^{2}\right) / \partial v_{\mathrm{ph}}$ respectively, are shown as the function of $v_{\mathrm{ph}}$ for $\mathrm{H}, \mathrm{He}$ and $\mathrm{HHe}(1: 1)$ with the same plasma parameters in case ' $a$ ', as well as the matched phase velocity corresponding to the peak $\mathrm{K}_{\mathrm{B}}$ at different $m$ for each ion composition. The $v_{\mathrm{ph}}$ and the dropping rate are normalized by thermal velocity of helium $v_{\text {thHe }}$ and $1 / v_{\text {thHe }}$, respectively. As expected, in HHe plasma, the matched $c_{\mathrm{S}, \mathrm{HHe}}$ is between the matched $c_{\mathrm{s}, \mathrm{H}}$ in single-species $\mathrm{H}$ plasma and $c_{\mathrm{s}, \mathrm{He}}$ in single-species He plasma, with $c_{\mathrm{s}, \mathrm{HHe}} / v_{\text {thHe }} \sim 4$ and $c_{\mathrm{s}, \mathrm{HHe}} / v_{\mathrm{thH}} \sim 2$. Because $c_{\mathrm{s}, \mathrm{HHe}}$ is closer to $v_{\mathrm{thH}}$, the Landau damping is mainly contributed by $\mathrm{H}$ ions, and the dropping rate of $\chi_{\mathrm{HHe}}^{\mathrm{I}}$ is closer to $\chi_{\mathrm{H}}^{\mathrm{I}}$ in case of single $\mathrm{H}$ ions, as shown in Fig. 10. However, due to the contributions of He ions, the dropping rate of $\left|1+\chi_{\mathrm{HHe}}\right|^{2}$ is larger than $\left|1+\chi_{\mathrm{H}}\right|^{2}$ of the single $\mathrm{H}$ case. As a consequence, the decrease of $\chi_{\mathrm{HHe}}^{\mathrm{I}}$ with $v_{\mathrm{ph}}$ is slower than the decrease of $\left|1+\chi_{\mathrm{HHe}}\right|^{2}$ in HHe plasma near $c_{\mathrm{s}, \mathrm{HHe}}$, which leads to $\partial \mathscr{D}^{\mathrm{I}} / \partial v_{\mathrm{ph}}<0$ and thus in regime I in case ' $a$ '.

In Fig. 9, the regime II is constrained between a limited range of $A_{\mathrm{m}} T_{\mathrm{e}} / T_{\mathrm{i}}$ when $T_{\mathrm{i}}\left(1-n_{\mathrm{e}} / n_{\mathrm{c}}\right) / n_{\mathrm{e}}$ is small, while it is totally regime I when $T_{\mathrm{i}}\left(1-n_{\mathrm{e}} / n_{\mathrm{c}}\right) / n_{\mathrm{e}}$ is large enough. This general feature of the partition between regime I and II can be explained as follows. When $T_{\mathrm{i}}\left(1-n_{\mathrm{e}} / n_{\mathrm{c}}\right) / n_{\mathrm{e}}$ is large enough, $-\mathscr{D}_{\text {ion }}^{\mathrm{I}}$ increases with $v_{\mathrm{ph}}$ for all possible $v_{\text {ph }}$ of IAW, as shown in Fig. 11(b). Since $-\mathscr{D}_{\mathrm{e}}^{\mathrm{I}}$ also increases (linearly) with $v_{\text {ph }}$ for $v_{\text {ph }} \ll v_{\text {the }},-\mathscr{D}^{\mathrm{I}}$ increases with $v_{\text {ph }}$ over the entire range of all possible
$c_{\mathrm{S}}$, rendering such $T_{\mathrm{i}}\left(1-n_{\mathrm{e}} / n_{\mathrm{c}}\right) / n_{\mathrm{e}}$ belonging to regime I for all $A_{m} T_{\mathrm{e}} / T_{\mathrm{i}}$. When $T_{\mathrm{i}}\left(1-n_{\mathrm{e}} / n_{\mathrm{c}}\right) / n_{\mathrm{e}}$ is smaller, as $v_{\text {ph }}$ increases, $-\mathscr{D}_{\text {ion }}^{\mathrm{I}}$ increases to a maximum firstly then decreases to a minimum, because $\chi_{\text {ion }}^{\mathrm{I}}$ drops with $v_{\mathrm{ph}}$ faster and faster after $v_{\mathrm{ph}}>v_{\mathrm{th} \alpha}$, and its dropping rate exceeds the dropping rate of $\left|1+\chi_{\text {ion }}\right|^{2}$ beyond this maximum point, as shown in Fig. 11(a). While the minimum point arises because $-\mathscr{D}$ ion rises sharply towards a peak at large $v_{\text {ph }}$ where $\chi_{\text {ion }}+1$ approaches zero. This peak corresponds to the greatest possible matched $c_{\mathrm{s}}$ when $A_{m} T_{\mathrm{e}} \gg T_{\mathrm{i}}$, and $\chi_{\mathrm{e}} \ll 1$ is negligible in the dispersion equation $\epsilon \approx 1+\chi_{\text {ion }}=0$. For a given $T_{\mathrm{i}}$, the matched $c_{\mathrm{S}}$ increases with the increased $A_{\mathrm{m}} T_{\mathrm{e}} / T_{\mathrm{i}}$, consequently, the ion damping decreases while the electron damping increases, as shown in Figs. 7(b) and 8(b). When $A_{\mathrm{m}} T_{\mathrm{e}} / T_{\mathrm{i}}$ is small enough, the contribution of $-\mathscr{D}$ ion dominates. The boundary coincides with the boundary when only the ion damping considered alone as shown in Fig. 9. The lower boundary corresponds to the maximum point of $-\mathscr{D}_{\mathrm{ion}}^{\mathrm{I}}$. For a parameter point with $A_{\mathrm{m}} T_{\mathrm{e}} / T_{\mathrm{i}}$ located below the lower boundary, the matched $c_{\mathrm{s}}$ is on the left side of the phase velocity corresponding to the maximum point of $-\mathscr{D}_{\text {ion }}^{\mathrm{I}}$, thus $-\mathscr{D}^{\mathrm{I}}$ increases with $v_{\mathrm{ph}}$ and this parameter point is in regime I. While for a parameter point with $A_{\mathrm{m}} T_{\mathrm{e}} / T_{\mathrm{i}}$ located above the lower boundary, the matched $c_{\mathrm{s}}$ is on the right side of the maximum point, thus $-\mathscr{D}^{\mathrm{I}}$ decreases with $v_{\mathrm{ph}}$ and this parameter point is in regime II. With the increase of $A_{\mathrm{m}} T_{\mathrm{e}} / T_{\mathrm{i}}$, the matched $c_{\mathrm{S}}$ becomes closer and closer to the minimum point of $-\mathscr{D}_{\mathrm{ion}}^{\mathrm{I}}$, but $-\mathscr{D}_{\mathrm{e}}^{\mathrm{I}}$ which increases with $v_{\text {ph }}$ becomes more and more important. At the upper right boundary of regime II with a relatively large $T_{\mathrm{i}}\left(1-n_{\mathrm{e}} / n_{\mathrm{c}}\right) / n_{\mathrm{e}}, c_{\mathrm{S}}$ reaches the minimum point before $-\mathscr{D}_{\mathrm{e}}^{\mathrm{I}}$ becomes sufficiently important. While for smaller $T_{\mathrm{i}}\left(1-n_{\mathrm{e}} / n_{\mathrm{c}}\right) / n_{\mathrm{e}}$, the increase of $-\mathscr{D}_{\mathrm{e}}^{\mathrm{I}}$ with $v_{\text {ph }}$ completely cancels the decrease of $-\mathscr{D}_{\text {ion }}^{\mathrm{I}}$ with $v_{\mathrm{ph}}$ before the phase velocity approaches the minimum point of $-\mathscr{D}$ ion as shown in Fig. 11(a), where the asterisk symbol indicates the location of the phase velocity corresponding to the minimum point of $-\mathscr{D}^{\mathrm{I}}$ with consideration of electron damping. This minimum point of $-\mathscr{D}^{\mathrm{I}}$ corresponds to the upper boundary of regime II at small $T_{\mathrm{i}}\left(1-n_{\mathrm{e}} / n_{\mathrm{c}}\right) / n_{\mathrm{e}}$ in Fig. 9 .

In high-Z plasmas, the Langdon effect can be more significant and the super-Gaussian exponent can approach about 5 . With the same definition of parameter regimes discussed above, the boundary between regime I and II is shown in Fig. 12 for $\mathrm{Au}$ and $\mathrm{AuB}$ plasma (including both $\mathrm{Au}^{10} \mathrm{~B}$ and $\left.\mathrm{Au}{ }^{11} \mathrm{~B}\right)$, respectively. Since the weak dependence of $-\mathscr{D}_{\mathrm{e}}^{\mathrm{I}}$ on $m$, the parameter boundary shifts with $m$ indistinctly as shown in Fig. 12(b). Here, only the boundary at $m=2$ is shown for Au plasma. It is noticed that when the ion damping is considered alone, the regime II of AuB plasma is enclosed in the regime II of Au plasma. In fact, this holds generally for mixing low$\mathrm{Z}$ species into the single ion species plasma with higher charge state. However, taking into account contributions from the electron damping, parameter region of regime II of $\mathrm{Au}$ plasma is reduced, and the regime II of $\mathrm{AuB}$ 

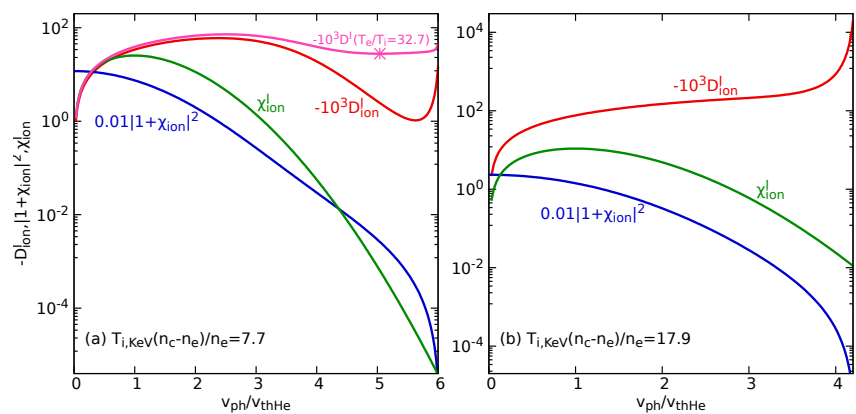

FIG. 11: The typical variation of $-\mathscr{D}_{\text {ion }}^{\mathrm{I}},\left|1+\chi_{\text {ion }}\right|^{2}$, and $\chi_{\text {ion }}^{\mathrm{I}}$ with $v_{\mathrm{ph}}$ for He plasma with $T_{\mathrm{i}}\left(1-n_{\mathrm{e}} / n_{\mathrm{c}}\right) / n_{\mathrm{e}}=7.7$ (a) and 17.9 (b). In the left panel, $\mathscr{D}^{\mathrm{I}}=\mathscr{D}_{\text {ion }}^{\mathrm{I}}+\mathscr{D}_{\mathrm{e}}^{\mathrm{I}}$ with $T_{\mathrm{e}} / T_{\mathrm{i}}=32.7$ corresponding to the upper boundary of regime II at $T_{\mathrm{i}}\left(1-n_{\mathrm{e}} / n_{\mathrm{c}}\right) / n_{\mathrm{e}}=7.7$ is also shown with its minimum point indicated by the asterisk symbol.

plasma locates inside the regime I of Au plasma. An interesting point of Fig. 12 is that the boundary of regime II for $\mathrm{Au}^{10} \mathrm{~B}$ plasma is upwardly shifted relative to that for $\mathrm{Au}{ }^{11} \mathrm{~B}$ plasma. Such an isotope effect should be embodied especially near the boundary of regime II. As shown in Fig. 12 case 'd' belongs to regime I in Au plasma, and spans both regime I and II in AuB plasma. Thus, as expected, the peak $\mathrm{K}_{\mathrm{B}}$ decreases with $m$ monotonically in $\mathrm{Au}$ plasma, while the trend of the peak $\mathrm{K}_{\mathrm{B}}$ with $m$ becomes non-monotonic and depends sensitively on the isotopic type of the low-Z species B in AuB plasma, as shown in Fig. 6.
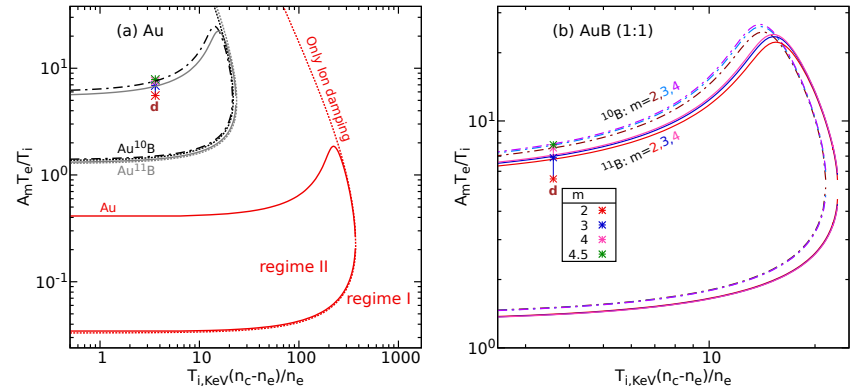

FIG. 12: (a) The boundary between regime I and II as specified by $A_{m} T_{\mathrm{e}} / T_{\mathrm{i}}$ versus $T_{\mathrm{i}}\left(n_{\mathrm{c}}-n_{\mathrm{e}}\right) / n_{\mathrm{e}}$ for $\mathrm{Au}$ (red solid line), $\mathrm{Au}^{10} \mathrm{~B}$ (black dot-dashed line) and $\mathrm{Au}^{11} \mathrm{~B}$ (gray solid line) plasma. The dotted curves show the boundary with ion damping considered only. (b) Zoomed in boundary for $\mathrm{Au}^{10} \mathrm{~B}$ (dot-dashed curves) and $\mathrm{Au}^{11} \mathrm{~B}$ (solid curves) plasma at different $m$. The asterisk symbols indicate the case 'd' with conditions specified in Table II, with the four points corresponding to $m=2, m=3, m=4$ and $m=4.5$ from bottom to top.

Using case 'd' as example, we take a closer look into the effects of the super-Gaussian EEDF and mixture near the upper boundary between regime I and II. In Au plasma, the electron damping dominates over the negligible ion damping since the matched $c_{\mathrm{s}}$ corresponding to the peak $\mathrm{K}_{\mathrm{B}}$ is much larger than ion thermal velocity $v_{\text {thAu }},-\mathscr{D}^{\mathrm{I}} \approx-\mathscr{D}_{\mathrm{e}}^{\mathrm{I}}$ increases with the matched phase velocity $c_{\mathrm{s}}$ and hence $\lambda_{\mathrm{B}}$, but decreases with $m$ as shown in Fig. 13(b). The first effect has a greater impact in case ' $\mathrm{d}$ ', which leads the peak $\mathrm{K}_{\mathrm{B}}$ decrease with $m$ as shown in Fig. 13(a). After mixing the low-Z boron species into $\mathrm{Au}$ plasma, the variation of the ion damping with $v_{\mathrm{ph}}$ due to $\mathrm{B}$ ions is not negligible compared to the variation of electron damping with $v_{\mathrm{ph}}$, as shown in Fig. 14(b). Furthermore, $-\mathscr{D}_{\text {ion }}^{\mathrm{I}}$ decreases with $\lambda_{\mathrm{B}}$ whereas $-\mathscr{D}_{\mathrm{e}}^{\mathrm{I}}$ increases with $\lambda_{\mathrm{B}}$. The trend of the peak $\mathrm{K}_{\mathrm{B}}$ with $m$ depends on the competition of the opposite change of $-\mathscr{D}_{\mathrm{e}}^{\mathrm{I}}$ and $-\mathscr{D}_{\text {ion }}^{\mathrm{I}}$ with $\lambda_{\mathrm{B}}$. In $\mathrm{Au}^{11} \mathrm{~B}$ plasma, this competition effect is the main reason for the non-monotonic change of the peak $K_{\mathrm{B}}$ with $m$. Nevertheless, the obviously high $-\mathscr{D}^{\mathrm{I}}$ at $m=2$ as shown in Fig. 14(a) leads the significant lower value of peak $\mathrm{K}_{\mathrm{B}}$ at $m=2$ than $m=3,4,5$ as shown in Fig. 6(b). Compared to ${ }^{11} \mathrm{~B}$ ion, the lighter ${ }^{10} \mathrm{~B}$ ion has a larger thermal velocity as shown in Fig. 14(b). As a result, near the peak wavelength, the ion damping contributed by ${ }^{10} \mathrm{~B}$ is larger than ${ }^{11} \mathrm{~B}$. On one hand, this leads to a smaller $\mathrm{K}_{\mathrm{B}}$ in $\mathrm{Au}^{10} \mathrm{~B}$ than in $\mathrm{Au}^{11} \mathrm{~B}$ plasma as shown in Fig. 6(b). On the other hand, it makes the contribution of $-\mathscr{D}$ i $\mathrm{I}$ to $-\mathscr{D}^{\mathrm{I}}$ become larger in $\mathrm{Au}^{10} \mathrm{~B}$ plasma, which leads to a stronger decreasing trend of $-\mathscr{D}^{\mathrm{I}}$ with $\lambda_{\mathrm{B}}$ as shown in Fig. 14(a). Thus, the peak $\mathrm{K}_{\mathrm{B}}$ increase with $m$ in $\mathrm{Au}^{10} \mathrm{~B}$ plasma as shown in Fig. 6(b).
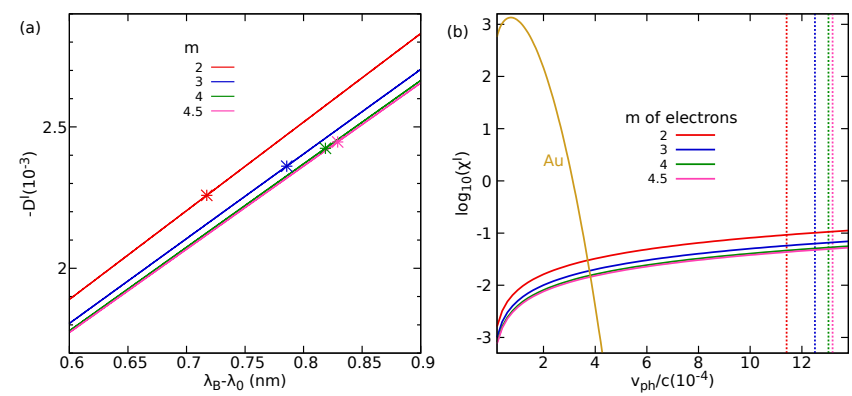

FIG. 13: (a) $-\mathscr{D}^{\mathrm{I}}$ (solid line) versus wavelength shift $\left(\lambda_{\mathrm{B}}-\lambda_{0}\right)$ for Au plasma at $m=2$ (red), $m=3$ (blue), $m=4$ (green) and $m=4.5$ (magenta). The asterisk symbols indicate the wavelength location corresponding to the peak $\mathrm{K}_{\mathrm{B}}$ in case 'd'. (b) $\chi_{\alpha}^{\mathrm{I}} \propto-\partial f_{\alpha}^{x} / \partial v_{x}$ of electrons at different $m$ and $\mathrm{Au}$ ions. The IAW phase velocities corresponding to the peak wavelength for each $m$ in case ' $d$ ' are indicated by the vertical dotted lines.

\section{DISCUSSION AND SUMMARY}

It is worthwhile to mention that in the high-density gas-filled hohlraum experiments on NIF facility, the peak wavelength of SRS is shorter than the simulated results obtained by the inline ray-tracing model and artificial seed of SRS backscattered light is needed to match the experimental reflectivity [11]. Commonly, the SRS of the inner cone is mainly stimulated in the high density region with $n_{\mathrm{e}}>0.1 n_{\mathrm{c}}$ and $T_{\mathrm{e}}<5 \mathrm{KeV}$ located in the 

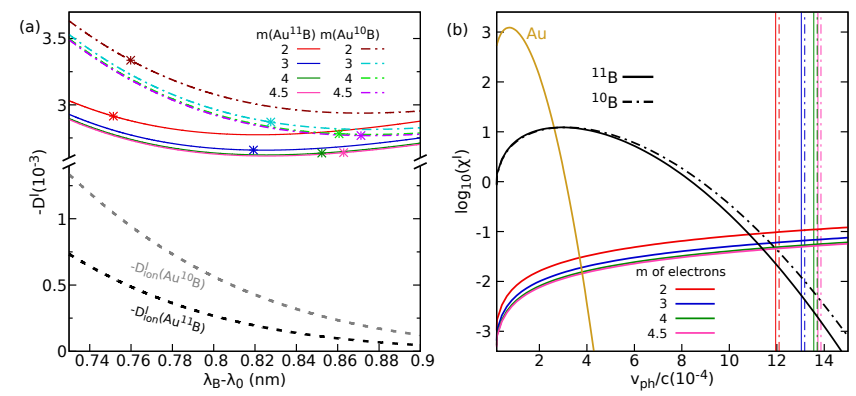

FIG. 14: (a) $-\mathscr{D}^{\mathrm{I}}$ (solid and dash-dotted lines) and $-\mathscr{D}_{\text {ion }}^{\mathrm{I}}$ (dashed line) versus wavelength shift $\left(\lambda_{\mathrm{B}}-\lambda_{0}\right)$ for $\mathrm{Au}^{10} \mathrm{~B}$ and $\mathrm{Au}^{11} \mathrm{~B}$ plasmas at different $m$. The asterisk symbols indicate the wavelength location corresponding to the peak $\mathrm{K}_{\mathrm{B}}$ at each $m$ in case 'd'. (b) $\chi_{\alpha}^{\mathrm{I}} \propto-\partial f_{\alpha}^{x} / \partial v_{x}$ of electrons at different $m$, Au ions, ${ }^{10} \mathrm{~B}$ ions, and ${ }^{11} \mathrm{~B}$ ions. The IAW phase velocities corresponding to the peak wavelength for each $m$ in case ' $\mathrm{d}$ ' are indicated by the vertical dot-dashed lines for $\mathrm{Au}^{10} \mathrm{~B}$ plasma and by the vertical solid lines for $\mathrm{Au}^{11} \mathrm{~B}$ plasma.

small $k_{1} \lambda_{\text {De }}$ regime where the wavelength of backscattered light of SRS decreases with the super-Gaussian exponent $m$. As a result, the simulated peak wavelength of SRS light is expected to be shorter with the consideration of the Langdon effect, which could be a possible explanation for this discrepancy in SRS spectra. Besides, considering the enhancement of SRS due to the Langdon effect in ray-tracing calculations may also ameliorate the current problem of the underestimated SRS reflectivity without the artificial seed. For SBS, the typical parameters $T_{\mathrm{e}} / T_{\mathrm{i}} \sim 1.4-6$ and $T_{\mathrm{i}} \sim \mathrm{KeV}$ in plasma ablated from hohlraum wall in experiments are roughly located in regime II of the high-Z plasma mixed with low-Z ions like $\mathrm{AuB}$, but located in regime I of the single high-Z plasma like Au. The Langdon effect itself can decrease the convective growth of SBS in single high-Z plasma but enhance SBS in mixed plasma, which may attenuate the improvement in suppression of SBS by mixing low-Z ion species into the high-Z plasma. Here, the influences of Langdon effect on SRS and SBS are mainly investigated based on the linear convective gain obtained from Vlasov model. In actual experiments, some possible nonlinear effects like electron trapping and the three-dimensional distribution of the high intensity speckles within the over- lapped laser beams make the coupling process of LPIs more complex, which still needs deeper investigations in future.

In summary, the Langdon effect should be prevalent in ICF hohlraum experiments and important to LPIs due to the sensitivity of LPIs on EEDF. Based on linear analysis, it is found that the peak wavelength of the scattered wave and peak value of the spatial growth rate of both SRS and SBS processes are observably influenced by the Langdon effect which induces a super-Gaussian EEDF. For SRS, a super-Gaussian EEDF modifies the dispersion relation of EPW, yielding a redshift or blueshift of the peak wavelength. However, the Landau damping of EPW is always reduced by the increased super-Gaussian exponent $m$. Consequently, the peak spatial growth rate of SRS always increases with $m$. For SBS, by reducing the low energy electron number to shield the ions, a superGaussian EEDF always increases the ion acoustic velocity, leading to a redshift of SBS scattered wavelength. However, the effects of a super-Gaussian EEDF on the peak spatial growth rate of SBS depend on the plasma condition such as electron density, electron temperature and ion temperature, and the ion composition (including the isotopic type) in a complex way. The boundary between different regimes of the enhancement or reduction of SBS by Langdon effect is given, and distinct behaviors are presented for low-Z and high-Z plasma with typical parameters in hohlraum plasma. The clarification of the Langdon effect on SRS and SBS can make us better understand the experimentally observed spectra and growth of SRS and SBS. Also it provides valuable references for improvement of the physical modeling and simulations of the LPI processes.

\section{ACKNOWLEDGMENTS}

This work was supported by the National Key R\&D Program of China (Grant No. 2017YFA0403204), the Science Challenge Project (Grant No. TZ2016005), the National Natural Science Foundation of China (Grant No. 11875093 and 11875091), and the Development Funds of CAEP (Grant No. CX20210040).
[1] A. Bruce Langdon. Nonlinear inverse bremsstrahlung and heated-electron distributions. Physical Review Letters, 44(9):575-579, 1980.

[2] J P Matte, M Lamoureux, C Moller, R Y Yin, J Delettrez, J Virmont, and T W Johnston. Non-Maxwellian electron distributions and continuum X-ray emission in inverse Bremsstrahlung heated plasmas. Plasma Physics and Controlled Fusion, 30(12):1665-1689, 1988.

[3] Su Ming Weng, Zheng Ming Sheng, and Jie Zhang. Inverse bremsstrahlung absorption with nonlinear effects of high laser intensity and non-Maxwellian distribution. Physical Review E - Statistical, Nonlinear, and Soft Matter Physics, 80(5):1-5, 2009.

[4] David Turnbull, Arnaud Colaïtis, Aaron M. Hansen, Avram L. Milder, John P. Palastro, Joseph Katz, Christophe Dorrer, Brian E. Kruschwitz, David J. Strozzi, and Dustin H. Froula. Impact of the Langdon effect on crossed-beam energy transfer. Nature Physics, 16:181-185, 2020.

[5] Jian Zheng, C. X. Yu, and Z. J. Zheng. Effects of non- 
maxwellian (super-gaussian) electron velocity distribution on the spectrum of thomson scattering. Physics of Plasmas, 4(7):2736-2740, 1997.

[6] A. L. Milder, H. P. Le, M. Sherlock, P. Franke, J. Katz, S. T. Ivancic, J. L. Shaw, J. P. Palastro, A. M. Hansen, I. A. Begishev, W. Rozmus, and D. H. Froula. Evolution of the electron distribution function in the presence of inverse bremsstrahlung heating and collisional ionization. Phys. Rev. Lett., 124:025001, 2020.

[7] John D. Lindl, Peter Amendt, Richard L. Berger, S. Gail Glendinning, Siegfried H. Glenzer, Steven W. Haan, Robert L. Kauffman, Otto L. Landen, and Laurence J. Suter. The physics basis for ignition using indirect-drive targets on the national ignition facility. Physics of Plasmas, 11(2):339-491, 2004.

[8] Liang Hao, Yiqing Zhao, Dong Yang, Zhanjun Liu, Xiaoyan $\mathrm{Hu}$, Chunyang Zheng, Shiyang Zou, Feng Wang, Xiaoshi Peng, Zhichao Li, Sanwei Li, Tao Xu, and Huiyue Wei. Analysis of stimulated raman backscatter and stimulated brillouin backscatter in experiments performed on sg-iii prototype facility with a spectral analysis code. Physics of Plasmas, 21(7):072705, 2014.

[9] D. J. Strozzi, E. A. Williams, D. E. Hinkel, D. H. Froula, R. A. London, and D. A. Callahan. Ray-based calculations of backscatter in laser fusion targets. Physics of Plasmas, 15(10):102703, 2008.

[10] G. N. Hall, O. S. Jones, D. J. Strozzi, J. D. Moody, D. Turnbull, J. Ralph, P. A. Michel, M. Hohenberger, A. S. Moore, O. L. Landen, L. Divol, D. K. Bradley, D. E. Hinkel, A. J. Mackinnon, R. P. J. Town, N. B. Meezan, L. Berzak Hopkins, and N. Izumi. The relationship between gas fill density and hohlraum drive performance at the national ignition facility. Physics of Plasmas, 24(5):052706, 2017.

[11] D. J. Strozzi, D. S. Bailey, P. Michel, L. Divol, S. M. Sepke, G. D. Kerbel, C. A. Thomas, J. E. Ralph, J. D. Moody, and M. B. Schneider. Interplay of laser-plasma interactions and inertial fusion hydrodynamics. Phys. Rev. Lett., 118:025002, 2017.

[12] V Yu Bychenkov, W Rozmus, and V T Tikhonchuk. Stimulated Raman scattering in non-Maxwellian plasmas. Physics of Plasmas, 4(5):1481, 1997.

[13] Bedros B. Afeyan, Albert E. Chou, J. P. Matte, R. P. J. Town, and William J. Kruer. Kinetic theory of electronplasma and ion-acoustic waves in nonuniformly heated laser plasmas. Phys. Rev. Lett., 80:2322-2325, 1998.

[14] P. Neumayer, R. L. Berger, L. Divol, D. H. Froula, R. A. London, B. J. MacGowan, N. B. Meezan, J. S. Ross, C. Sorce, L. J. Suter, and S. H. Glenzer. Suppression of stimulated brillouin scattering by increased landau damping in multiple-ion-species hohlraum plasmas. Phys. Rev. Lett., 100:105001, 2008.

[15] P. Neumayer, R. L. Berger, D. Callahan, L. Divol, D. H. Froula, R. A. London, B. J. MacGowan, N. B. Meezan, P. A. Michel, J. S. Ross, C. Sorce, K. Widmann, L. J. Suter, and S. H. Glenzer. Energetics of multiple-ion species hohlraum plasmas. Physics of Plasmas, 15(5):056307, 2008.

[16] J. F. Drake, P. K. Kaw, Y. C. Lee, G. Schmid, C. S. Liu, and Marshall N. Rosenbluth. Parametric instabilities of electromagnetic waves in plasmas. The Physics of Fluids, 17(4):778-785, 1974.

[17] Francis F Chen. Introduction to plasma physics and controlled fusion, volume 1. Springer, 1984.

[18] R. K. Kirkwood, J. D. Moody, J. Kline, E. Dewald, S. Glenzer, L. Divol, P. Michel, D. Hinkel, R. Berger,
E. Williams, J. Milovich, L. Yin, H. Rose, B. Macgowan, O. Landen, M. Rosen, and J. Lindl. A review of laser-plasma interaction physics of indirect-drive fusion. Plasma Physics and Controlled Fusion, 55(10):103001, 2013.

[19] E. A. Williams, R. L. Berger, R. P. Drake, A. M. Rubenchik, B. S. Bauer, D. D. Meyerhofer, A. C. Gaeris, and T. W. Johnston. The frequency and damping of ion acoustic waves in hydrocarbon (ch) and two-ion-species plasmas. Physics of Plasmas, 2(1):129-138, 1995.

[20] Q.S. Feng, Z.J. Liu, L.H. Cao, C.Z. Xiao, L. Hao, C.Y. Zheng, C. Ning, and X.T. He. Interaction of parametric instabilities from $3 \omega$ and $2 \omega$ lasers in large-scale inhomogeneous plasmas. Nuclear Fusion, 60(6):066012, 2020.

[21] In fact, it can be proven under the condition $\partial \mathscr{D}^{\mathrm{I}} / \partial v_{\mathrm{ph}}=$ 0 and $\mathscr{D}^{\mathrm{R}}=0, \mathrm{~K}_{\mathrm{B}}$ given in Eq. (15) satisfies $\partial \mathrm{K}_{\mathrm{B}} / \partial \omega_{s}=$ 0 . That is, the peak condition of $K_{B}$ is exactly satisfied. So when the weak dependence of $-\mathscr{D}_{\mathrm{e}}^{\mathrm{I}}$ on $m$ can be neglected, these two conditions give the exact conditions for the boundary where the trend of the peak $K_{B}$ with $m$ changes.

\section{Appendix A: Proof that the ion acoustic velocity is monotonically increasing with $A_{m} T_{\mathrm{e}}$}

The ion acoustic velocity is given by Eq. (19) as $c_{\mathrm{s}}^{2}=$ $(A \pm \sqrt{B}) / 2$, with the plus for the fast mode, and the minus for the slow mode. Firstly we define

$s_{\mathrm{e}} \equiv \frac{A_{m} T_{\mathrm{e}}}{\bar{Z} M_{\mathrm{p}} q}=\frac{A_{m} T_{\mathrm{e}}}{\bar{Z} M_{\mathrm{p}}\left(1+A_{m} k^{2} \lambda_{\mathrm{De}}^{2}\right)} \propto \frac{A_{m} T_{\mathrm{e}}}{1+\epsilon_{0} k^{2} A_{m} T_{\mathrm{e}} / n_{\mathrm{e}} e^{2}}$,

It can be seen $s_{\mathrm{e}}$ is monotonically increasing with $A_{m} T_{\mathrm{e}}$. On the other hand,

$$
\frac{\partial A}{\partial s_{\mathrm{e}}}=\overline{Z^{2} / A}>0
$$

and

$\frac{\partial \sqrt{B}}{\partial s_{\mathrm{e}}}=\frac{1}{\sqrt{B}}\left[\left(\frac{\gamma_{1}}{A_{1}}-\frac{\gamma_{2}}{A_{2}}\right)\left(\frac{f_{1} Z_{1}^{2}}{A_{1}}-\frac{f_{2} Z_{2}^{2}}{A_{2}}\right) \frac{T_{\mathrm{i}}}{M_{\mathrm{p}}}+s_{\mathrm{e}}\left(\overline{Z^{2} / A}\right)^{2}\right]$

Using Eqs. (21), (A2) and (A3), we obtain

$B\left(\frac{\partial A}{\partial s_{\mathrm{e}}}\right)^{2}-\left(\sqrt{B} \frac{\partial \sqrt{B}}{\partial s_{\mathrm{e}}}\right)^{2}=\left[2 Z_{1} Z_{2}\left(\frac{\gamma_{1}}{A_{1}}-\frac{\gamma_{2}}{A_{2}}\right) \frac{T_{\mathrm{i}}}{M_{\mathrm{p}}}\right]^{2} \frac{f_{1} f_{2}}{A_{1} A_{2}} \geq 0$

Therefore,

$$
\frac{\partial A}{\partial s_{\mathrm{e}}} \geq\left|\frac{\partial \sqrt{B}}{\partial s_{\mathrm{e}}}\right|
$$

In other words,

$$
\frac{\partial c_{\mathrm{s}}^{2}}{\partial s_{\mathrm{e}}}=\frac{1}{2}\left[\frac{\partial A}{\partial s_{\mathrm{e}}} \pm \frac{\partial \sqrt{B}}{\partial s_{\mathrm{e}}}\right] \geq 0
$$

So $c_{\mathrm{s}}$ is monotonically increasing with $s_{\mathrm{e}}$, which is itself monotonically increasing with $A_{m} T_{\mathrm{e}}$. 


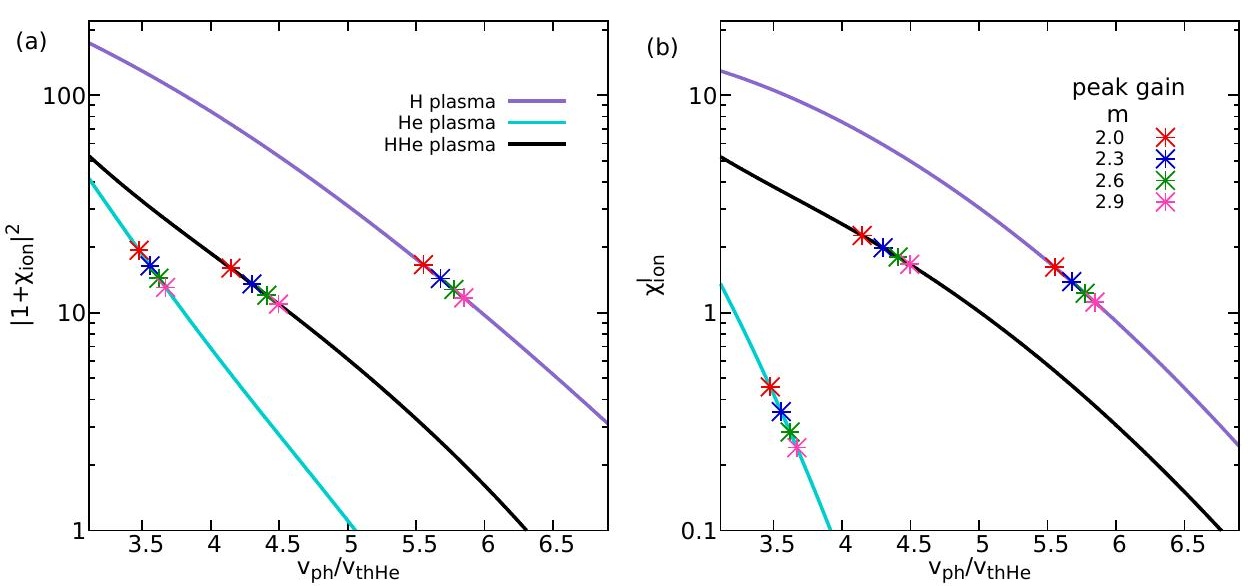

